\begin{abstract}
HICKS, KEVIN ERVIN. Highlighting Validity and Placement of Risk Information in Drug Manufacturers' Direct-to-Consumer Web Pages. (Under the direction of Michael S. Wogalter.)

Since the mid-1980s, direct-to-consumer (DTC) advertising has changed the way a pharmaceutical company markets prescription medications. DTC advertising has steadily become an integral way of communicating information about prescription drugs to end users and research has shown that the Internet is growing as a potential source of drug information. The current study examined the effects of colored highlighting validity (Valid, Invalid and No highlighting) and placement (Top, Middle and Bottom) of target risk keywords in web pages using two kinds of visual search tasks. The two tasks were the same except that in one a target was always present and in the other the target was either present or absent. For both tasks dependent variables were response time and accuracy. The results indicate that valid highlighting significantly reduced response time and increased accuracy across both tasks. Invalid highlighting was not significantly different compared to no highlighting for both tasks in terms of response time or accuracy. Results for both tasks also showed that placement of target keywords on a web page had a significant effect. Top and middle placements significantly reduced response time and increased accuracy compared to placement at the bottom. In general highlighting has benefits when the sought information is validly highlighted but has no cost when non-target information is highlighted instead. The present findings confirm the results found in studies in other domains. Implications for potential application of highlighting and placement and for future research are discussed.
\end{abstract}


Highlighting Validity and Placement of Risk Information in Drug Manufacturers' Direct-toConsumer Web Pages

by

Kevin Ervin Hicks

A thesis submitted to the Graduate Faculty of

North Carolina State University in partial fulfillment of the requirements for the Degree of

Master of Science

Psychology

Raleigh, North Carolina

2007

APPROVED BY:

Robert S. Dicks III

Sharolyn Converse-Lane

Michael S. Wogalter

Chair of Advisory Committee 


\section{BIOGRAPHY}

Kevin E. Hicks currently resides in Raleigh, NC where he has lived and worked for the past eight years. Mr. Hicks moved to Raleigh from Charleston, WV where he was born. His father Earl still lives there while his mother Linda lives with his only brother in Raleigh.

He graduated from Nitro High School in 1993 and attended Marshall University to pursue a degree in Psychology. After attaining his Bachelors of Arts in Psychology, he moved to Raleigh to pursue a graduate degree at North Carolina State University. For the

past six years, Kevin has worked in the Cognitive Ergonomics Laboratory in the Psychology Department under the direction of Dr. Michael Wogalter. Currently, Kevin is employed by IBM developerworks as a Human Factors Engineer where he has worked on the design and testing of web sites and software for the last five years. 


\section{ACKNOWLEDGEMENTS}

I would first like to thank Dr. Michael Wogalter (committee chair) for his support, guidance, and providing me the opportunity to work with him in his lab doing different types research. Secondly, I would like to thank Dr. Sharolyn Converse-Lane and Dr. Stan Dicks (committee members) for all of their patience and support during this process.

I would also like to give thanks to my friend Adriel Boals for his help, especially with my final analysis in SAS. In addition I would also like to give thanks to Kimberly Brantley for being a friend and my mentor at IBM.

I would like to give thanks to my best friend, brother and mentor, Bill for providing me with his programming knowledge, ingenuity, and overall guidance during this process. Additionally I would like to give thanks to my sister-in-law Leigh Jay for her love, friendship, and support. Also special thanks to my parents Earl and Linda for the years of continuous support, encouragement, friendship, and love that has meant a lot to me.

Finally, I would finally like to thank Alison Planthaber for her encouragement and support during my many years in graduate school. I appreciate everything you have done for me. 


\section{TABLE OF CONTENTS}

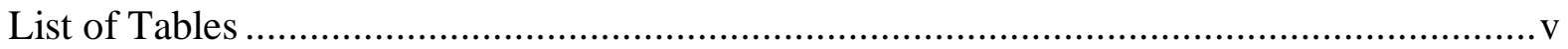

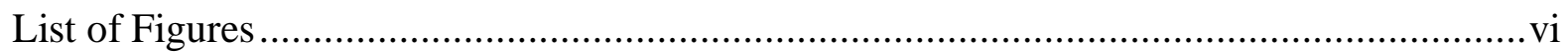

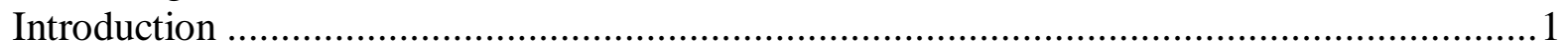

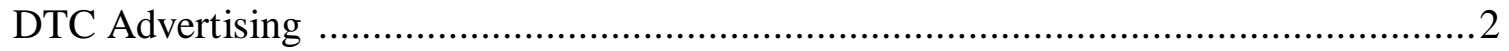

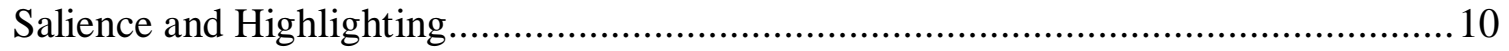

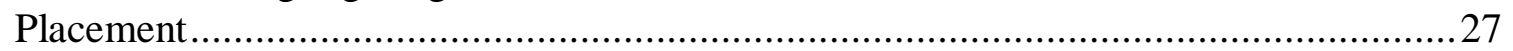

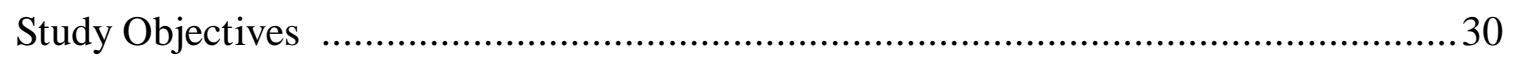

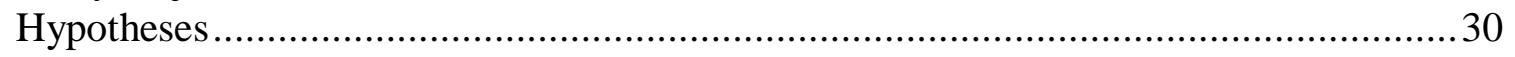

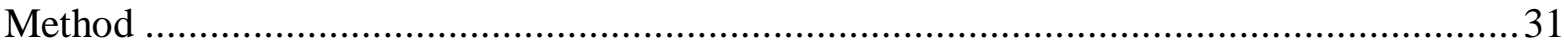

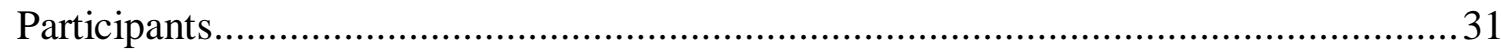

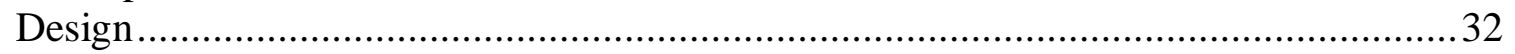

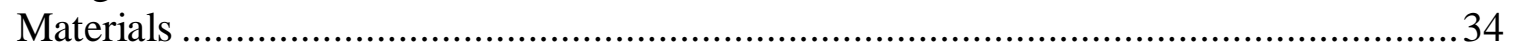

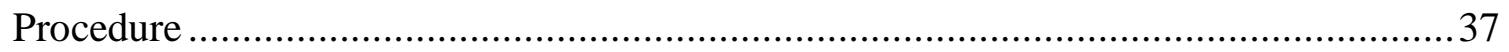

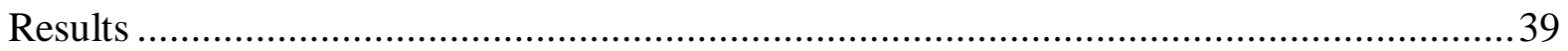

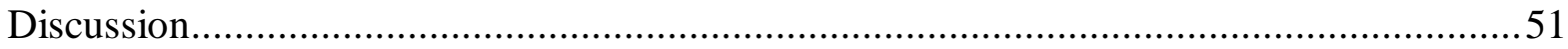

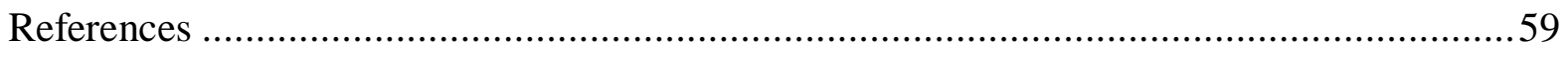

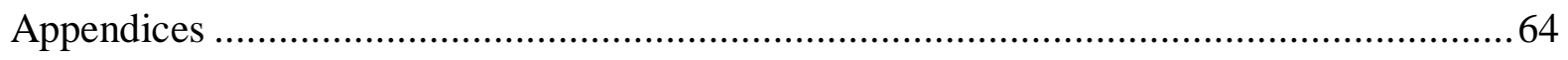

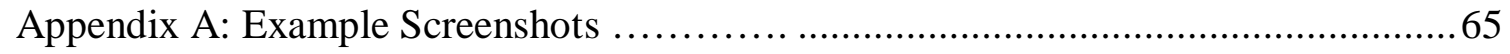

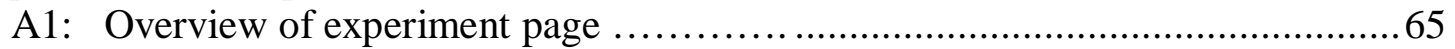

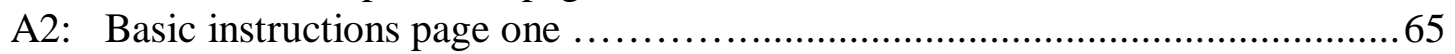

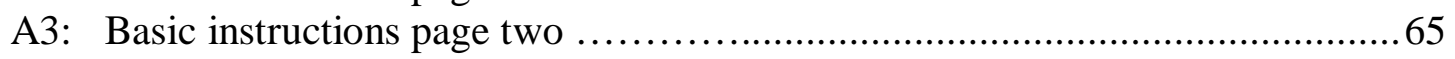

A4: Demonstration page (Target always present task)....................................66

A5: Demonstration page (Target present/absent task) ....................................66

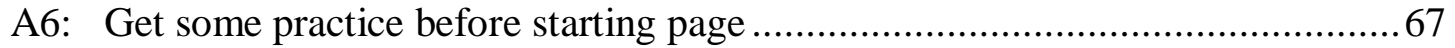

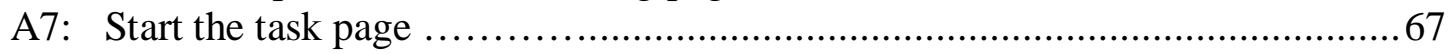

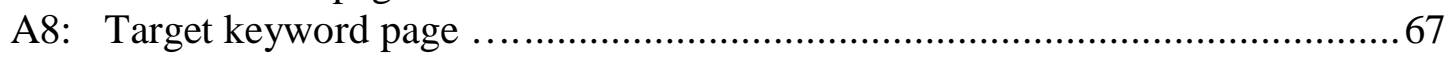

A9: Target always absent task - Risk page (Wellbutrin) ...................................6 68

A10: Target always absent task - Risk page (Advair) .........................................69

A11: Target present/absent task - Risk page (Wellbutrin) .................................. 70

A12: Target present/absent task - Risk page (Advair) .................................... 71

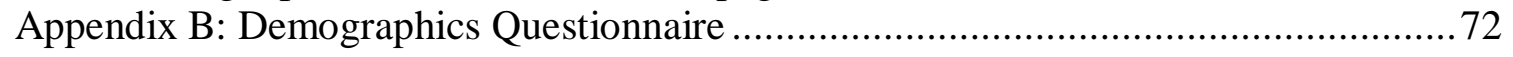

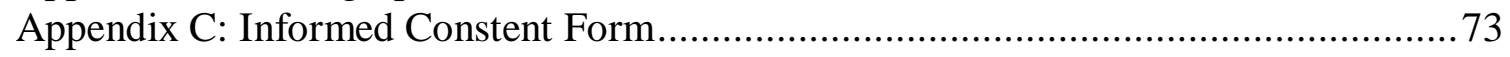

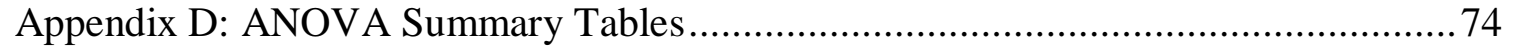




\section{LIST OF TABLES}

Table 1. List of keywords used as targets for both tasks.............................. 36

Table 2. Gender and race demographics...........................................40

Table 3. Age demographics............................................................40

Table 4. Experience with computers and Internet demographics data..................40

Table 5. Response time: Target always present task $\mathrm{x}$ highlighting x placement..........43

Table 6. Accuracy: Target always present task x highlighting x placement ..............44

Table 7. Response time: Target present/absent task x highlighting x placement..........46

Table 8. Response time: Target present/absent task interactions.....................47

Table 9. Accuracy: Target present/absent task x highlighting x placement ..............49

Table 10. Accuracy: Target present/absent task interactions $\ldots \ldots \ldots \ldots \ldots \ldots \ldots \ldots \ldots \ldots . .50$ 


\section{LIST OF FIGURES}

Figure 1. Communication Human Information Processing (C-HIP) model..............19

Figure 2. Target always present task web page model...................................33

Figure 3. Target present/absent task web page model..................................34

Figure 4. Response time: Validity x Placement (Target always present task)..............43

Figure 5. Accuracy: Validity x Placement (Target always present task).....................45

Figure 6. Response time: Validity x Placement (Target present/absent task)...............48

Figure 7. Accuracy: Validity x Placement (Target present/absent task)......................50 


\section{INTRODUCTION}

Since the mid-1980s, direct-to-consumer (DTC) advertising has changed the way a pharmaceutical company market prescription medications. In the past, prescription drug manufacturers have directed their advertisements to trained health care professionals, rather than the end user. Through DTC advertising, manufacturers of prescription drugs market their products directly to the end user, and the overall amount of advertising has steadily increased since its inception (Wilkes, 2000).

DTC advertisements can include several different types of media including: print ads, television and radio ads, and the World Wide Web (WWW). DTC advertising has steadily become an integral way of communicating information about prescription drugs (Holmer, 1999). Research (Everett, 1991) suggests that consumers want to be informed about the benefits and risks associated with their medications. Through these advertising mediums drug companies market their products as well as communicate benefits and risks information to potential consumers.

DTC advertisements are different from most other advertising as a consumer must go through an intermediary, by requiring a physician to give a prescription (Calfee, 2002). DTC advertisements are also somewhat unique in that they are regulated by the U.S. Food and Drug Administration (FDA). The FDA has regulations that manufacturers must give drug risk information that is accessible and clear among the other content contained in DTC advertisements. 
The objective of the current study was to explore ways of facilitating the acquisition of risk information within online DTC manufacturers' advertising web pages. The present research focused on the potential effects of highlighting and placement of risk information in DTC web sites. First, a literature review of DTC advertising regulations, as well as a review of relevant research using DTC advertisements is presented. Then, a review of relevant risk communication and human computer interaction research (HCI) examined the effect of highlighting validity and placement of important information in different environments including the web.

\section{Direct-to-Consumer Advertising}

With the increase of DTC prescription drug marketing, the FDA responded with regulations for broadcast (television and radio) and print media (newspaper, magazine and billboards) DTC advertisements (FDA, 1997, 1999). These regulations established four basic requirements for all DTC advertisements, these include: (1) brief summary, (2) fair balance,

(3) major statement, and (4) adequate provision. The "brief summary" is a short listing of all the side effects and contraindications associated with the prescription drug. The "fair balance" requirement is that DTC advertisements must have an equal presentation of both the benefits and risks associated with the drug. The "major statement" requirement states that broadcast DTC ads must communicate side effects and contraindications. Finally, the regulations state that, if a broadcast advertisement does not provide a brief summary, it must 
include an "adequate provision" in its place. The "adequate provision" requirement is that a manufacturer must provide convenient access to the prescription drug's approved labeling.

These FDA regulations specify that the major statement, along with the adequate provision requirements, can provide all the necessary information for risk disclosure to consumers. Primarily, adequate provision serves as a more complete source of risk information, Giving the full disclosure of risk information for the prescription drug by (1) disclosing that a pharmacist or physician can be contacted for further information, (2) tollfree number, (3) reference to a print advertisement, or (4) World Wide Web (WWW) address. The toll-free number is so consumers can call to request the drug's package labeling. The print advertisement may be used as a source of complete product information / labeling for the drug advertised. The WWW address could potentially provide another means of distributing labeling information in a Portable Document Format (PDF) and on the web pages themselves.

Overall these FDA regulations are viewed as just a set of general guidelines for manufacturers using DTC advertising. This allows manufacturers an increased amount of flexibility in the manner in which they provide risk and safety information to potential consumers. To date only a few studies have been published examining the factors affecting risk presentation and most of them have been with print or broadcast DTC advertisements.

Research by Wogalter, Paine, Mills, and Smith-Jackson (1999) examined the use of DTC print advertisements, focusing on whether integrated or separated benefit and risk information was more effective. Different versions of a mock magazine were designed with 
the conditions: (a) separated, no color; (b) separate, color; (c) integrated, no color; (d) integrated, color; (e) separated, enhanced; and (f) control of DTC ads with each created for six fictional prescriptions drugs. The drug advertisements were merged into a set of realisticappearing magazine pages. Each participant saw all six-drug advertisements, but in different conditions. The results showed that greater knowledge of risks is obtained, from a printed ad when risk information is simultaneously made more salient and placed in a separate location from the benefit information.

Another study, using DTC prescription drug television commercials, investigated different visual and auditory presentations of risk information (Kalsher and Wogalter, 2006). FDA allows risks to be presented either aurally only or both visually and aurally. Kalsher and Wogalter compared the potential effectiveness of different combinations of audio and visual presentations on recall and recognition of incidentally presented risk information. Results showed that concurrent visual/audio risk disclosure produced the highest recall and recognition compared to the two modalities given separately. Analysis indicated that visual presentation yielded higher recall and recognition compared to risks presented only aurally. These findings suggest that current FDA standards of allowing aural risk information to be presented to consumers in television DTC prescription drug advertisements is not optimal. More specifically, aural only risk presentation was found as the least efficient; which is the current standard practice for television DTC prescription drug advertisements.

In addition, the FDA conducted two telephone surveys examining consumer awareness for print and television DTC advertising along with subsequent related consumer 
behavior (Aikin, 2002; FDA, 1999). Each survey consisted of about a thousand randomly selected individuals who had visited a doctor within the last three months. Results indicated that, $50 \%$ of the respondents of the 1999 survey and $43 \%$ in the 2002 survey reported, that after being exposed to some form of DTC advertisement they looked for additional product information on the drug advertised. Results of the 2004 survey also showed that safety information was rated as the highest priority when searching for additional information with $61 \%$ reported looking for side effects, $17 \%$ drug interaction information, and $13 \%$ dangers associated with drugs. The majority of respondents in both surveys reported being exposed to DTC prescription drug television advertisements followed closely by magazine ads. The results also give some indication of the increasing importance of the Internet as a source of prescription drug information.

Additional research by Bell, Kravitz, and Wilkes (1999) evaluated individuals' awareness, attitudes, misconceptions, and behavioral responses towards DTC prescription drug advertisements. Half of the respondents thought that DTC prescription drug advertisements required governmental approval. Additionally, $43 \%$ reported thinking that only safe drugs could be advertised in DTC ads. The results indicate the potential for other misconceptions about the governmental regulation of DTC prescription drug advertisements.

In the recent past consumers have had limited to use of more traditional sources of prescription drug information (i.e., physician and pharmacist). Increasingly consumers are gaining access to other non-traditional sources of prescription drug information. Some more recent additions to these sets sources of prescription drug information include: the WWW 
(e.g., manufacturer's product and second party informational web sites), manufacturer's consumer phone number, medical reference texts, and friend or family. These sources can vary greatly in terms of information presentation (e.g., amount and type of content, usability, amount of interactivity). These differences may influence their use by consumers, in regards to their expectations to get answers.

Hicks et al. (2005) evaluated nine potential sources including: pharmacist, physician, friend/family, manufacturer's web site, second-party web site (WebMD), manufacturer's phone number, medical reference text, television ads, and print ads. Participants were asked to rate each source of information according to three perception/belief dimensions: (a) relative likelihood-of-use, (b) perceived ease-of-use, and (c) perceived completeness of the information provided. The results show that pharmacists and physicians are the two most preferred sources of prescription drug information. Manufacturers' DTC prescription drug web sites were consistently rated as one of top potential sources of information on all three rating dimensions compared to the other sources. Also results show that two of the four methods of adequate provision (toll-free number and print advertisements) were consistently rated among the lowest across all three sets of ratings, with exception of pharmacist/physician. The most preferred adequate provision was manufacturer's web site.

FDA research has shown an increasing interest among consumers in the Internet as source of prescription drug information (Aikin, 2002; FDA, 1999). As a more recent addition to DTC marketing campaigns, the Internet poses new difficulties in effective risk disclosure. The WWW offers several potential advantages (e.g., convenience, customization) to people 
seeking information. However, it has some disadvantages, such as being more complex to use and by making certain information less accessible than other information (e.g., requiring more clicks and scrolling). The FDA regulations have specific stipulations for the layout and placement of risk information in print advertisements (warnings, precautions, negative side effects, and contraindications), but it has no specific regulations for the WWW (FDA, 1999). Current guidelines for drug advertising on manufacturer's web sites are based on advertising regulations for broadcast and print media. Issues associated with balanced information on the Internet are more complex due to the fact that it is a different medium with its own characteristics. Web sites are constructed of pages at different levels of a web site hierarchy. This capability is not present in broadcast and print media. Where the information is placed in the hierarchy affects the search time and number of clicks a user must make. For example, research has shown that with each additional click, information is less likely to be found (Vigilante \& Wogalter, 2005). The potential of manufacturers placing risks further away from benefits could result in users never viewing the risk information.

"Balanced" benefit and risk information is difficult to define for all media, but it is even more difficult for the Internet. For television and print advertisements "balance" is defined as presenting an equal number of benefit and risks. Web sites are usually comprised of multiple pages of varying types of content (e.g., HTML, PDF, multimedia) typically organized into a hierarchical structure forming its basic information architecture. The information architecture of the web site determines how the web site may be organized and establishes its labels used for navigation. Moreover a web site's information architecture has 
a great influence in how a web site is navigated (Nielsen, 2000). Users of web sites have to navigate this hierarchy to find the benefit and risk information when they are not presented on the homepage. This fundamental difference in the manner in which information is presented in web site advertisements as compared with TV and print ads has created an interest in determining how risks and benefits are actually being presented in DTC drug ads on the WWW.

A recent study examined a random sample of DTC prescription drug advertisements on the WWW to determine how manufacturers place benefit and risk information in relation to the home page and to each other (Hicks et al., 2005). The study consisted of two-sample time periods (March 1999, July 2003) that measured the number of clicks required to reach the risk and benefit information from the homepage and from each other. Also examined was whether scrolling was required to view the risk or benefit information. Lastly, also determined was the need for a separate program, Portable Document Format (PDF) file reader, to view the risk information. The results suggest that the risks are being placed at deeper levels ("more distant") of the web site hierarchy than the benefit information. Risk information is more difficult to access than benefit information by requiring significantly more clicks and the need to scroll compared to benefits. Additionally $20 \%$ of the web sites required the user to view the risk information in PDF format adding another layer of inaccessibility.

Research has shown that risk information placed deeper in hierarchical structure is less likely to be found (Vigilante \& Wogalter, 2005). This study had benefit and risk 
information manipulated in different ways, within 2 DTC manufacturer prescription drug advertisements web sites to see their effect on whether a user can locate a drugs' risk and benefit information. The benefit and risk information presented were either integrated together or separated, and placed on same page or on different pages within the web site's hierarchy. The results also suggested that information placement can affect the likelihood that risk and benefit information will be noticed and subsequently read. The results indicated that people have trouble finding risk information at lower levels of a web site hierarchy. Risk information placed at three clicks or more from the home page may not be found. The results also indicate that the placement of the risk information can affect the likelihood that the benefit information is noticed, read, and remembered. Results also suggest that risk information should ideally be placed on a separate second level page with a prominent link at the top of the navigation bar. Additionally, benefit information is found more often, found faster and with less clicks when presented on the home page.

Research investigating methods of increasing the likelihood that risk information is noticed within WWW DTC drug ads does not seem to exist. The current study seeks to examine important factors that influence noticeability and effective communication of risk information on the web.

The next two sections will draw upon relevant research from risk and warnings communication and human-computer interaction (HCI) research to consider potential methods to enhance WWW DTC advertisements. Both research domains have a substantial amount of research, which has examined ways of facilitating communication of information. 
Risk communication research has primarily focused on effective communication of warning and risk information in different environments (home, work). HCI work has researched methods to help facilitate searching for information in visual displays. Each of the following sections will address the effect of highlighting/salience and placement on attracting attention and visual search performance.

\section{Salience and Highlighting}

Wogalter and Leonard (1999) offer a series of recommendations that have been shown to facilitate attention capture. Including attention capturing features such as: highlighting, color, contrast, and placement of the warning can increase a warning's noticeability and its overall effectiveness (Barlow \& Wogalter, 1993; Frantz \& Miller, 1993; Young \& Wogalter, 1990; Wogalter, Kalsher, \& Racicot, 1993 and Wogalter et al., 1987)

Research by Young (1991) examined several ways of increasing the noticeability of warnings. The presentation of four salience variables was manipulated including: (1) pictorial, (2) signal icon, (3) border and (4) color. Participants were shown 96 simulated alcohol labels on a computer. Half of the trials participants were shown a warning in the simulated labels and for the half no warning was present. Results show that warnings printed in red text were found significantly faster that those in black text. Kline et al. (1993) examined the impact of color on warnings research and found a significant main effect for color, again showing that the presence of color can affect the readability and salience of warnings. 
Warnings in products manuals are an important means of communicating safety information related to products. They can provide information about safely operating the equipment without imposing an injury. Consumers do not always read or keep products manuals so fast, effective warning communication is essential (Wogalter et al., 1991). Warnings in products manuals need to be designed to facilitate warning comprehension and to enhance recall of related hazards. Young and Wogalter (1990) conducted two experiments that manipulated the use of icons and conspicuous print to measure their effect on comprehension and memory of products warnings. Both experiments were identical with the exception of the products and product manuals used. Four variations of instruction manual were created for each experiment. Each manual composed of one experimental manipulation of the product warning which contained either: (1) conspicuous print and icon, (2) conspicuous print only, (3) icon only, or (4) conspicuous print or icon not presented. Conspicuous print was shown as 18-point Times, bolded with color for highlighting compared to the other background text (12 point Helvetica). Three different tests were used to measure comprehension and memory of the warnings. Results showed that participants who received the manual with conspicuous print icons had significantly better comprehension and recall of product warnings than compared to the other three versions. Also more specifically comprehension was greater for conspicuous print versus plain print. Results further suggest the use of making text more conspicuous versus the surrounding text. Conspicuous or salient warnings are more likely to attract readers' attention because they are not as likely to blend into the surrounding pictures and text (Wogalter et al., 1991). 
Use of conspicuous print has also been shown to be beneficial in alcoholic beverage warnings in magazine and television advertisements (Barlow \& Wogalter, 1993). One experiment involved the manipulation of warnings in a magazine and the second television advertisements. Participants that viewed the print ads with warnings with highly conspicuous print retained significantly more risk information than those who viewed less conspicuous print. Also less conspicuous print was found to be no better than no warnings.

Human-computer interaction research. Visual search research is one of the most relevant paradigms in relation to the proposed study. Visual search behavior in displays is often not consistent and can be internally driven but there are some factors that tend to guide the allocation of attention (Wickens \& Hollands, 2000). An advantage of color highlighting is to draw attention to a particular location in order to aid in a visual search. Highlighting can make visual search more effective by causing users to switch from a serial to a parallel visual search (Matlin, 2002). Thus, highlighting can be a highly effective cue for identification and selection of relevant objects in a visual search task.

Tullis (1984) developed a model of visual search that predicted usability of information displayed in alphanumeric displays. The model uses four display characteristics: overall density, local density, grouping, and layout to predict user performance in extracting information. Much of the research performed on visual search performance has focused on the four same display characteristics used in Tullis' work. 
Research with visual displays has examined ways of facilitating visual search performance with the use of highlighting. Typically, highlighting is used to draw a user's attention to a certain area or even a particular word in the visual display. The main purpose of highlighting in displays is to facilitate visual search performance by capturing and directing the attention of the user to some relevant information. Specifically, in the case of text a common form of increasing salience is through the use of highlighting which has been shown to have the ability to draw attention of users when it is clearly visible.

Research using a cost-benefit analysis has been employed by researchers to measure the use of color highlighting when it is randomly predictive of relevant information (Jonides \& Mack, 1984). Response time and errors are used to infer measurement of the underlying cognitive processes. The basic premise for using a cost benefit analysis is that a highlighted invalid target could inadvertently be processed over a non-highlight valid target. This would thereby increase the likelihood of incorrect responses as well as increase response time to locate valid targets. This type of research takes into account validity of the correct item/target being identified using two types of visual search tasks, identification and classification. Participants performing an identification task are asked to locate a target within a set of distracters. In a classification task participants ask to determine whether a target is present or absent

Previous researchers have offered some conflicting support for the efficacy of highlighting. This early research with highlighting had primarily investigated various forms of highlighting (e.g., color, bulleting, blinking, reverse video, boxing) in alphanumeric visual 
display terminals (VDT). Gomberg (1985) investigated the use of highlighting in visual displays indicated that highlighting could have a negative effect on visual search. Participants instructed to search for a single target digit among a background of four distracter digits. Three highlighting conditions (boxing, blinking and reverse video) and one non-highlighted condition were included with only one digit being highlighted per trial. Results showed that search times were significantly longer for each of the three highlighting conditions compared to the control condition. These results seem counter-intuitive with the use highlighting.

Tan and Fisher (1989) sought to clarify this paradox of highlighting in visual displays. Two issues were raised in reaction to Gomberg's study. First of all, they addressed the issue that Gomberg did not use a more optimal form of highlighting (i.e. color). Secondly, they examined whether the use of valid highlighting for only half of the trials could further diminish any benefit of highlighting. Tan and Fisher's (1989) first experiment used color, reverse video, and blinking as highlighting methods compared to each other and to a non-highlighting condition. Results of the first experiment confirmed that alternate forms of highlighting can have different effects on visual search performance. The color highlighting condition produced significantly better performance than both the blinking and reverse video conditions, however there was no difference compared to the non-highlighted condition.

The second experiment was essentially same as the first with the exception that the highlighting conditions were always valid. Highlighting was considered "valid" if the target participants were searching for was the stimuli highlighted in the display. Results show that a 
participant's expectancy of highlighting validity is a major factor in determining performance. Results indicated that by maintaining $100 \%$ highlighting validity at $100 \%$ that even invalid highlighting could be useful.

Visual search studies have shown that stimuli differing in color from a homogeneous background appears to pop out and can be detected easily by observers (Treisman \& Gelade, 1980) The study showed that when an item is highlighted with color and valid participants found it quicker, thus illustrating the potential benefits of highlighting valid items with color. Participants, in this case, develop a strategy of shifting their attention to color highlighted items over non-highlighted items. These results indicate that even invalid highlighting can facilitate visual search if a more optimal form of highlighting such as color, is used. Additional research with color highlighting in VDT displays has shown that color can be an effective cue for the identification and selection of relevant objects (Martin, McDonald \& Patton, 1987). It is also a prevalent method of emphasizing important information in a visual display.

Martin et al.'s (1987) research using an identification task sought to examine the effect of different types of highlighting in visual displays. Participants were to identify one of four potential single digit targets in a linear array of five single digit numbers. Results showed that valid trials were located fastest with invalid and no highlighting trials being equally slower. Results also showed that color highlighting has some benefit without a cost in visual search. From this research Martin et al. coined the phrase the color advantage effect. 
However, research using a classification task (Jonides \& Yantis; 1984, 1990) has shown that this is not always the case that color highlighting has benefit without a cost in a visual search task. Using similar conditions to Martin et al.'s research they sought to examine if features such as color and abrupt onset can force attention to particular item on a visual display. Results showed that abrupt onset produce an attention shift whereas, color highlighting trial showed no differences between valid and invalid trials.

Both sets of research sought to examine why highlighting can force attention to a particular item/target that has no higher probability of being a valid or correct target than any other item/target being shown in a visual display. However, they did not find the same effect of benefit without any cost using colored highlighting. Additional research by Eustace et al. (2000) with VDTs sought to clarify if the limits of the color advantage effect, using both an identification and classification task. Results showed that the color advantage effect may be task dependent where a target has to be identified (identification task) versus when it may or may not be present (classification task).

A major consideration of this previous research has been the use of small alphanumeric screens, commonplace at the time. These findings might be viewed as somewhat unrepresentative and may not be generalizable to the larger and increasingly more complex displays in use today. Donner et al. (1991) conducted a study that utilized a more complex display. Utilizing a display taken from a space shuttle they examined the effect of display format (existing vs. reformatted) and highlighting validity (valid, invalid, and no highlighting) on search performance. The results show that highlighting in more complex 
displays can be beneficial. There was a significant benefit to valid highlighting and cost to invalid highlighting with the existing display; however the reformatted version had no effect for highlighting. Again results suggest that the use of highlighting can be beneficial with virtually no cost for invalid highlighting in complex displays. Appropriate highlighting such as color has been shown to provide greater benefit compared to other types such as blinking, larger text, and boxing.

Although there is research from both the risk communication and HCI domains, it is limited regarding the presentation of warnings on the Internet. One study compared the use of a physical versus an online presentation of a toy's product warning (Santos \& Resnick, 2002). Participants were asked to select an appropriate toy for a 3 year old from one of eight choices. Four of the choices had a small parts hazard warning and the other four did not. Participants were asked to reference whatever materials were available to make the appropriate choice. The salience of the Internet warning was manipulated: high salience (red, signal word, outlined) versus low salience (embedded in product description present with the same black text and font type). Results showed that participants were more likely to notice the online warning versus the one physically printed on the toy's packaging. These findings also showed that significantly more risks were recalled for online versus physical warning presentation. Results showed that a warning placed on the Internet, can be an effective means to communicate risk information. Results also showed that increasing salience of a warning could positively affect whether it is noticed on a web page. 
Specifically, web usability research has shown that the majority of users quickly visually scan a new page, versus read word-for-word (Nielsen, 2000). Nielsen recommends the use of highlighting to make the page more scannable and to make important keywords catch the user's eye. Users rarely read web pages word-for-word due to the large amount of information on web pages a typical user may encounter (Price \& Price, 2002). Users are more likely to scan web page content, because it is a more efficient strategy versus reading serially. 


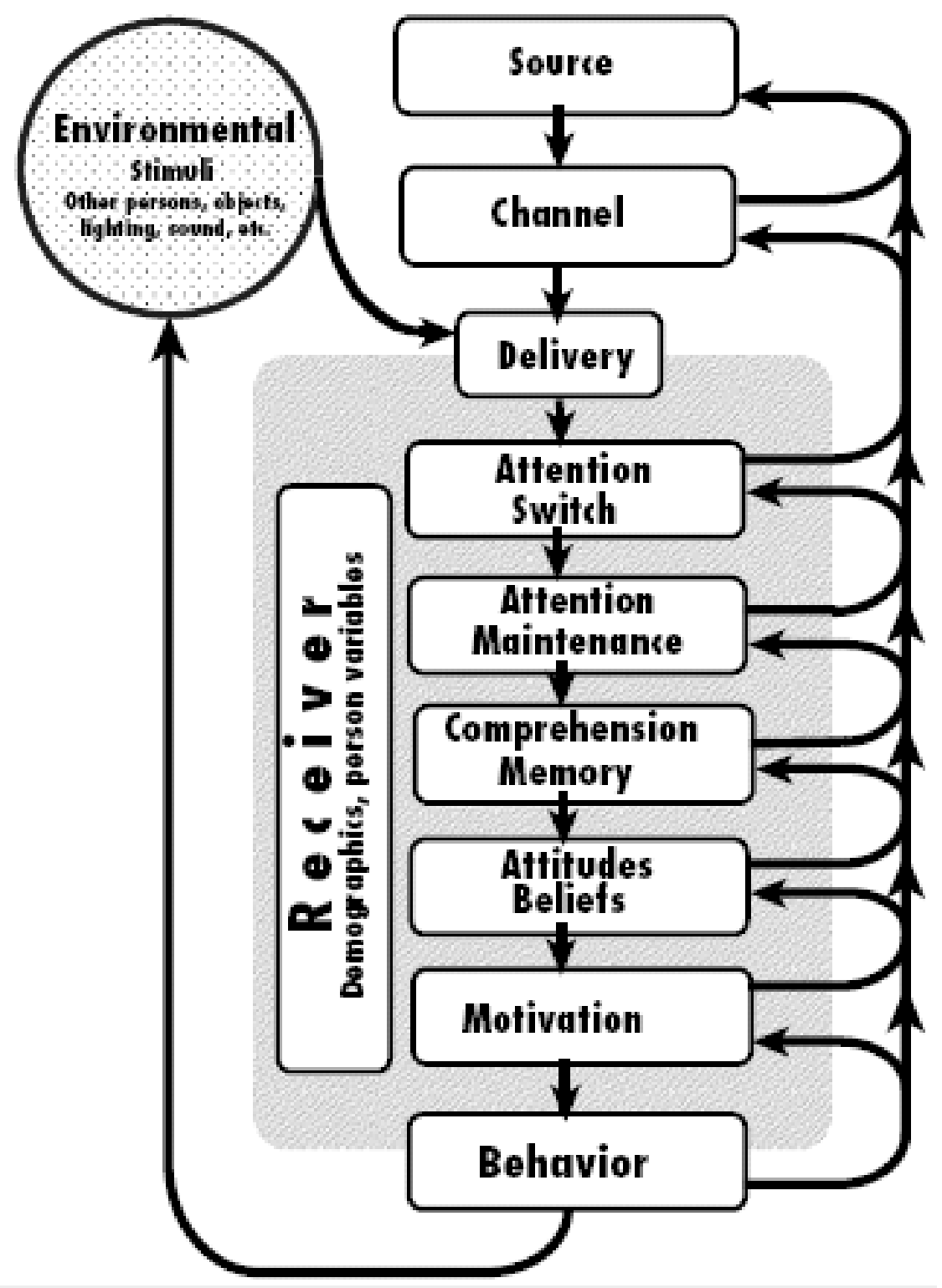

Figure 1. Communication Human Information Processing (C-HIP) model

Wogalter and Leonard (1999) offer a series of recommendations that have been shown to facilitate attention capture. Including attention capturing features such as: highlighting, color, contrast, and placement of the warning can increase a warning's 
noticeability and its overall effectiveness (Barlow \& Wogalter, 1993; Frantz \& Miller, 1993; Young \& Wogalter, 1990; Wogalter, Kalsher, \& Racicot, 1993 and Wogalter et al., 1987)

Research by Young (1991) examined several ways of increasing the noticeability of warnings. The presentation of four salience variables was manipulated including: (1) pictorial, (2) signal icon, (3) border and (4) color. Participants were shown 96 simulated alcohol labels on a computer. Half of the trials participants were shown a warning in the simulated labels and for the half no warning was present. Results show that warnings printed in red text were found significantly faster that those in black text. Kline et al. (1993) examined the impact of color on warnings research and found a significant main effect for color, again showing that the presence of color can affect the readability and salience of warnings.

Warnings in products manuals are an important means of communicating safety information related to products. They can provide information about safely operating the equipment without imposing an injury. Consumers do not always read or keep products manuals so fast, effective warning communication is essential (Wogalter et al., 1991). Warnings in products manuals need to be designed to facilitate warning comprehension and to enhance recall of related hazards. Young and Wogalter (1990) conducted two experiments that manipulated the use of icons and conspicuous print to measure their effect on comprehension and memory of products warnings. Both experiments were identical with the exception of the products and product manuals used. Four variations of instruction manual were created for each experiment. Each manual composed of one experimental manipulation 
of the product warning which contained either: (1) conspicuous print and icon, (2) conspicuous print only, (3) icon only, or (4) conspicuous print or icon not presented. Conspicuous print was shown as 18-point Times, bolded with color for highlighting compared to the other background text (12 point Helvetica). Three different tests were used to measure comprehension and memory of the warnings. Results showed that participants who received the manual with conspicuous print icons had significantly better comprehension and recall of product warnings than compared to the other three versions. Also more specifically comprehension was greater for conspicuous print versus plain print. Results further suggest the use of making text more conspicuous versus the surrounding text. Conspicuous or salient warnings are more likely to attract readers' attention because they are not as likely to blend into the surrounding pictures and text (Wogalter et al., 1991). Use of conspicuous print has also been shown to be beneficial in alcoholic beverage warnings in magazine and television advertisements (Barlow \& Wogalter, 1993). One experiment involved the manipulation of warnings in a magazine and the second television advertisements. Participants that viewed the print ads with warnings with highly conspicuous print retained significantly more risk information than those who viewed less conspicuous print. Also less conspicuous print was found to be no better than no warnings.

Human-computer interaction research. Visual search research is one of the most relevant paradigms in relation to the proposed study. Visual search behavior in displays is often not consistent and can be internally driven but there are some factors that tend to guide 
the allocation of attention (Wickens \& Hollands, 2000). An advantage of color highlighting is to draw attention to a particular location in order to aid in a visual search. Highlighting can make visual search more effective by causing users to switch from a serial to a parallel visual search (Matlin, 2002). Thus, highlighting can be a highly effective cue for identification and selection of relevant objects in a visual search task.

Tullis (1984) developed a model of visual search that predicted usability of information displayed in alphanumeric displays. The model uses four display characteristics: overall density, local density, grouping, and layout to predict user performance in extracting information. Much of the research performed on visual search performance has focused on the four same display characteristics used in Tullis' work.

Research with visual displays has examined ways of facilitating visual search performance with the use of highlighting. Typically, highlighting is used to draw a user's attention to a certain area or even a particular word in the visual display. The main purpose of highlighting in displays is to facilitate visual search performance by capturing and directing the attention of the user to some relevant information. Specifically, in the case of text a common form of increasing salience is through the use of highlighting which has been shown to have the ability to draw attention of users when it is clearly visible.

Research using a cost-benefit analysis has been employed by researchers to measure the use of color highlighting when it is randomly predictive of relevant information (Jonides $\&$ Mack, 1984). Response time and errors are used to infer measurement of the underlying cognitive processes. The basic premise for using a cost benefit analysis is that a highlighted 
invalid target could inadvertently be processed over a non-highlight valid target. This would thereby increase the likelihood of incorrect responses as well as increase response time to locate valid targets. This type of research takes into account validity of the correct item/target being identified using two types of visual search tasks, identification and classification. Participants performing an identification task are asked to locate a target within a set of distracters. In a classification task participants ask to determine whether a target is present or absent

Previous researchers have offered some conflicting support for the efficacy of highlighting. This early research with highlighting had primarily investigated various forms of highlighting (e.g., color, bulleting, blinking, reverse video, boxing) in alphanumeric visual display terminals (VDT). Gomberg (1985) investigated the use of highlighting in visual displays indicated that highlighting could have a negative effect on visual search. Participants instructed to search for a single target digit among a background of four distracter digits. Three highlighting conditions (boxing, blinking and reverse video) and one non-highlighted condition were included with only one digit being highlighted per trial. Results showed that search times were significantly longer for each of the three highlighting conditions compared to the control condition. These results seem counter-intuitive with the use highlighting.

Tan and Fisher (1989) sought to clarify this paradox of highlighting in visual displays. Two issues were raised in reaction to Gomberg's study. First of all, they addressed the issue that Gomberg did not use a more optimal form of highlighting (i.e. color). Secondly, they examined whether the use of valid highlighting for only half of the trials 
could further diminish any benefit of highlighting. Tan and Fisher's (1989) first experiment used color, reverse video, and blinking as highlighting methods compared to each other and to a non-highlighting condition. Results of the first experiment confirmed that alternate forms of highlighting can have different effects on visual search performance. The color highlighting condition produced significantly better performance than both the blinking and reverse video conditions, however there was no difference compared to the non-highlighted condition.

The second experiment was essentially same as the first with the exception that the highlighting conditions were always valid. Highlighting was considered "valid" if the target participants were searching for was the stimuli highlighted in the display. Results show that a participant's expectancy of highlighting validity is a major factor in determining performance. Results indicated that by maintaining $100 \%$ highlighting validity at $100 \%$ that even invalid highlighting could be useful. An additional study by Fisher et al. (1989) found that even at $25 \%$ and $75 \%$ highlighting validity, color highlighting had significantly better performance than a non-highlighted condition.

Visual search studies have shown that stimuli differing in color from a homogeneous background appears to pop out and can be detected easily by observers (Treisman \& Gelade, 1980) The study showed that when an item is highlighted with color and valid participants found it quicker, thus illustrating the potential benefits of highlighting valid items with color. Participants, in this case, develop a strategy of shifting their attention to color highlighted items over non-highlighted items. These results indicate that even invalid highlighting can 
facilitate visual search if a more optimal form of highlighting such as color, is used. Additional research with color highlighting in VDT displays has shown that color can be an effective cue for the identification and selection of relevant objects (Martin, McDonald \& Patton, 1987). It is also a prevalent method of emphasizing important information in a visual display.

Martin et al.'s (1987) research using an identification task sought to examine the effect of different types of highlighting in visual displays. Participants were to identify one of four potential single digit targets in a linear array of five single digit numbers. Results showed that valid trials were located fastest with invalid and no highlighting trials being equally slower. Results also showed that color highlighting has some benefit without a cost in visual search. From this research Martin et al. coined the phrase the color advantage effect.

However, research using a classification task (Jonides \& Yantis; 1984, 1990) has shown that this is not always the case that color highlighting has benefit without a cost in a visual search task. Using similar conditions to Martin et al.'s research they sought to examine if features such as color and abrupt onset can force attention to particular item on a visual display. Results showed that abrupt onset produce an attention shift whereas, color highlighting trial showed no differences between valid and invalid trials.

Both sets of research sought to examine why highlighting can force attention to a particular item/target that has no higher probability of being a valid or correct target than any other item/target being shown in a visual display. However, they did not find the same effect of benefit without any cost using colored highlighting. Additional research by Eustace et al. 
(2000) with VDTs sought to clarify if the limits of the color advantage effect, using both an identification and classification task. Results showed that the color advantage effect may be task dependent where a target has to be identified (identification task) versus when it may or may not be present (classification task).

A major consideration of this previous research has been the use of small alphanumeric screens, commonplace at the time. These findings might be viewed as somewhat unrepresentative and may not be generalizable to the larger and increasingly more complex displays in use today. Donner et al. (1991) conducted a study that utilized a more complex display. Utilizing a display taken from a space shuttle they examined the effect of display format (existing vs. reformatted) and highlighting validity (valid, invalid, and no highlighting) on search performance. The results show that highlighting in more complex displays can be beneficial. There was a significant benefit to valid highlighting and cost to invalid highlighting with the existing display; however the reformatted version had no effect for highlighting. Again results suggest that the use of highlighting can be beneficial with virtually no cost for invalid highlighting in complex displays. Appropriate highlighting such as color has been shown to provide greater benefit compared to other types such as blinking, larger text, and boxing.

Although there is research from both the risk communication and HCI domains, it is limited regarding the presentation of warnings on the Internet. One study compared the use of a physical versus an online presentation of a toy's product warning (Santos \& Resnick, 2002). Participants were asked to select an appropriate toy for a 3 year old from one of eight 
choices. Four of the choices had a small parts hazard warning and the other four did not. Participants were asked to reference whatever materials were available to make the appropriate choice. The salience of the Internet warning was manipulated: high salience (red, signal word, outlined) versus low salience (embedded in product description present with the same black text and font type). Results showed that participants were more likely to notice the online warning versus the one physically printed on the toy's packaging. These findings also showed that significantly more risks were recalled for online versus physical warning presentation. Results showed that a warning placed on the Internet, can be an effective means to communicate risk information. Results also showed that increasing salience of a warning could positively affect whether it is noticed on a web page.

Specifically, web usability research has shown that the majority of users quickly visually scan a new page, versus read word-for-word (Nielsen, 2000). Nielsen recommends the use of highlighting to make the page more scannable and to make important keywords catch the user's eye. Users rarely read web pages word-for-word due to the large amount of information on web pages a typical user may encounter (Price \& Price, 2002). Users are more likely to scan web page content, because it is a more efficient strategy versus reading serially.

\section{Placement}

Risk communication and HCI research have also examined the effect that placement has on communication of important information. Both have examined various kinds of placements of information within different visually complex environments. Relevant research 
in regards to placement for both risk communication and HCI domains is described in the following sections.

Risk communication research. Using a task analytic approach, Frantz and Rhoades (1993) had participants complete a set of tasks that included the unpacking and arranging of office furniture and equipment. A file cabinet was specifically included because of a common tipping hazard involved with overloading top drawers and the associated warning placed on the cabinet's shipping container. Warning placement on the file cabinet included: (1) on its shipping carton, (2) on top and bottom of file cabinet, (3) placed in front with drawers shut, and (4) placed on cardboard bridge in the top drawer. The third and fourth conditions were specifically designed to physically interfere with the loading of the file cabinet task assigned to participants. Overall results showed that careful placement of warnings can affect product use. Participants significantly noticed and read warning when it interfered within the task. Task interference conditions also yielded significantly greater behavioral compliance than non-interfering conditions. Results again indicate that a warning should be placed where it is more likely to be seen by intended users.

Wogalter, Kalsher, and Racicot (1993) also found that the placement of warning could significantly effect behavioral compliance. In a series of three studies they manipulated the placement of a warning that included: (1) within task instructions, (2) posted on a wall, and (3) no warning presented. Overall results of the studies consistently showed that by integrating a warning within the instructions of a task produced significantly higher behavioral compliance than the placement of the same warning on a nearby wall or when no 
warning was presented. Also warnings embedded in instructions can also influence the likelihood they will be noticed.

Strawbridge (1986) investigated the potential influence of a warning's placement and the use of highlighting can have on its detection. Results showed that the placement of the warning showed to have a significant positive effect on noticeability of a warning. Also highlighting could significantly increase the likelihood that an individual would read a warning.

Human-computer interaction research. Previous usability research has examined the effect of placement on finding information. Web usability research, in particular, has shown that information located further down on a web page is less likely to be seen than information located at the top of a web page (Lynch \& Horton, 1999; Nielsen, 2000; Spool et al., 1999). A basic convention in web designers is to display any important information so that is initially visible on the screen without scrolling.

Usability research by Jacob Nielsen has also shown that users often decide whether to stay or leave a web page based on what they can see without scrolling (Nielsen, 2000). Furthermore, Vigilante and Wogalter (2005) found that risk information is also less likely to be found if a user is required to scroll down a web page to view the information.

To date no research has sought to examine the effect of validity of color highlighting as well as the placement of risk information on web pages having safety information about prescription drugs. 


\section{Study Objectives}

The present study sought to determine the effect of highlighting validity and placement of target keywords within DTC web pages on response time and accuracy using two visual search tasks. Each task was representative of the typical kind of behavior a user might exhibit while examining a web page: (a) where users know what they are looking for is on the web page (target always present task), and (b) where users are not sure whether what they are looking for is on the web page, may be present or absent (target present/absent task).

\section{Hypotheses}

Based on the foregoing background description the following hypotheses were made. Hypothesis 1: Target keywords in the valid highlighting conditions will be found significantly faster and will be more accurate than target keywords in the invalid highlighting conditions.

Hypothesis 2: Target keywords in the valid highlighting conditions will be found significantly faster and will be more accurate than target keywords in the no highlighting conditions. 
Hypothesis 3: Target keywords in the no highlighting conditions will be found significantly faster and will be more accurate than target keywords in the invalid highlighting conditions.

Hypothesis 4: Target keywords placed in the top and middle of a web page will be found significantly faster than target keywords placed at the bottom of a web page independent of highlighting validity.

Additional exploratory analyses will be described in the Results section.

\section{METHOD}

\section{Participants}

The study consisted of 30 participants for each of the 2 visual search tasks for a total of 60 participants. Fifty percent of the participants were recruited from the introductory psychology course participant pool at North Carolina State University and the remaining 50 percent were recruited from the local community in Raleigh, NC. Some demographic data about the participant sample are presented in the Results section (Tables 2, 3 and 4). 
Design

The experimental design was a 3 Highlighting validity (Valid, Invalid and No Highlighting) x 3 Placement (Top, Middle and Bottom) x 2 visual search tasks (target always present and target present/absent). In the target always present task, participants performed a forced choice visual search task where they had to decide which of three paragraphs contained the target keyword on a web page. With the target present/absent task participants had to determine if a target was present or absent on a web page with the target randomly being present in $50 \%$ of the trials and absent for the other $50 \%$ (with randomized presentation). Because the tasks were different, the highlighting validity $\mathrm{x}$ placement variables were examined separately by task.

Dependent variables for both tasks included response time and accuracy which was defined as the proportion of correct responses. Analysis for the target present/absent task required a target to be present to examine the effects of highlighting validity and placement on response time and accuracy. Therefore, the target present/absent task ( 72 trials) required twice the number of trials per participant as the Target always present task (36 trials).

Highlighting validity represented three levels of conditions including: valid, invalid, and no highlighting. Highlighting was valid if the highlighted keyword was the target. Highlighting was invalid if a distracter keyword rather than the target keyword was highlighted. No highlighting conditions contained no highlighting on the web page.

Placement of target keywords was manipulated by placing it randomly within one of the three paragraphs of risk information text presented on a risk web page. 
Figures 2 and 3 present a simplified graphical representation/model of the risk web page for each task. The target keywords placed in the top and middle paragraph were always initially visible when the page loaded and therefore did not require scrolling. However, the bottom paragraph text required scrolling to make it visible on the screen.

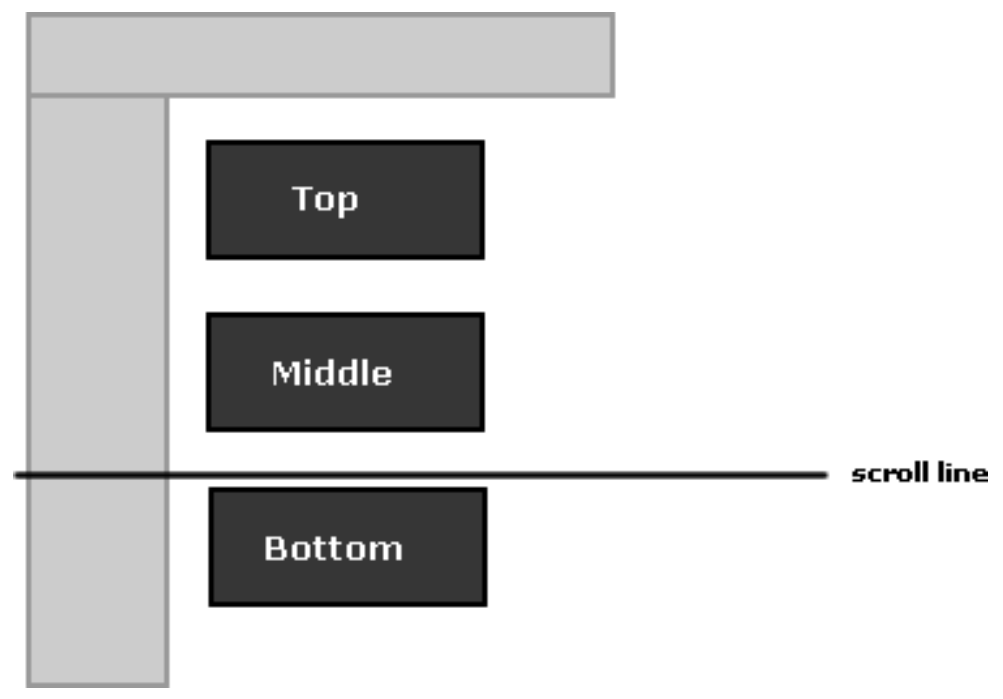

Figure 2. Target always present task web page model. In this representation the target keyword would be located anywhere within 1 of the 3 paragraphs (Top, Middle and Bottom). 


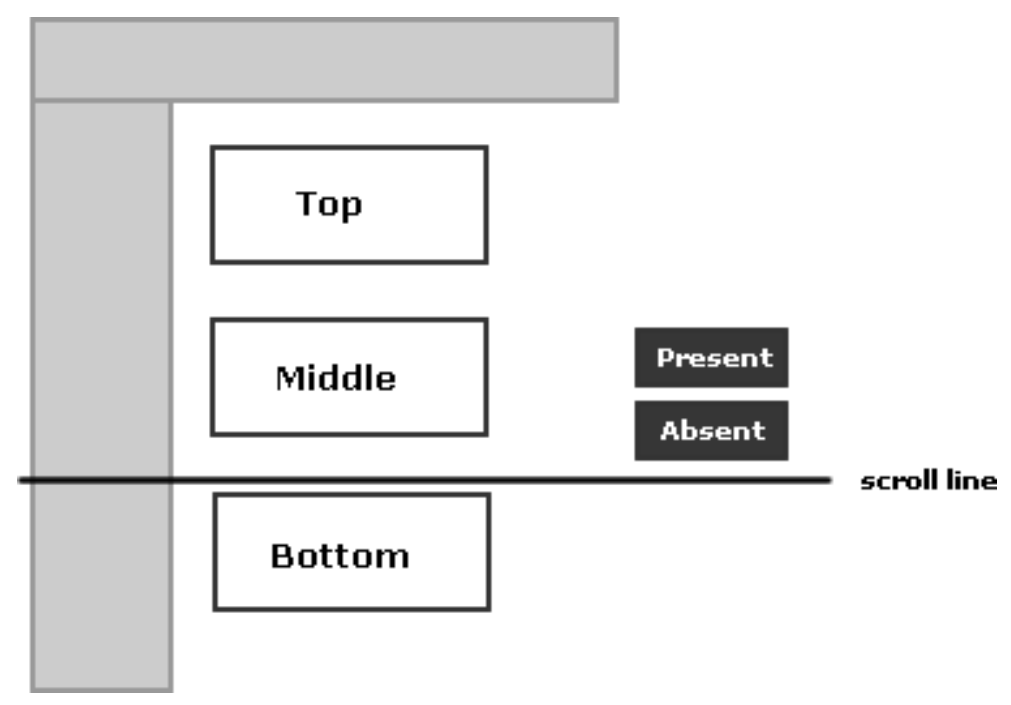

Figure 3. Target present/absent task model. In this representation the target keyword could present anywhere within 1 of the 3 paragraphs (Top, Middle and Bottom) or it may be absent from the page.

\section{Materials}

All study materials were presented in an Internet Explorer 6.0 web browser on a PC running Windows XP. The screen size and resolution were consistently presented on a 15inch $(23.3 \mathrm{~cm})$ diagonal LCD monitor, set at $1024 \times 768$ dpi resolution with the browser window maximized. Participants used an optical mouse to respond during the experiment.

Instruction pages. The instruction pages consisted of five portions including: 1) basic instructional pages, 2) demonstration page, 3) start practice trial page, 4) practice trials and 5) start the task page. Screenshots of the instruction pages are provided in Appendix A. The basic instructional for the experiment included a short overview page and two pages of task instructions (A1, A2 and A3). The overview page (A1) contained text that included: 1) Go thru some basic instructions, 2) View a short demonstration of the task, 3) Get some practice before starting, 4) Start the task (20-25mins) and 5) a link labeled 'Next'. Page 1 of the task 
instructions (A2) contained text that included: 1) The basic task is to locate a keyword on a web page, 2) Please concentrate on locating the keyword correctly please do not hurry and 3) a link labeled 'Next'. Page 2 of the task instructions (A3) contained text that included: 1) The mouse is the only thing you need to use for the entire task, 2) Important: Please do not use the back button and 3) a link labeled 'Next'.

The visual demonstration page consisted of 2 different versions with the only difference being the instructional text and the movie presented on the page. The target always present task demonstration page text included: 1. Obtain keyword, 2. Find keyword, 3. Click on the keyword and 4. Repeat (A4). Target present/absent task demonstration page text included: 1. Obtain keyword, 2. Find keyword, 3. Click on present or absent and 4. Repeat (A5). Each task's demonstration page also consisted of a short movie that automatically started playing when the page was loaded. The movie was a pre-recorded screen capture of an individual's browser as he or she completed a set of three practice trials. The screen capture was recorded using Camtasia (Techsmith) software and then it was saved as an AVI video formatted movie file so it could be played within the Internet Explorer (Microsoft) browser using a standard Windows media player browser plug in. The demonstration page also included a link labeled 'Play again" to reload the page / restarted the movie (prerecorded screen capture) and link labeled 'Next'.

The start practice trials page (A6) contained text that included: 1) Try it out before beginning the experiment and 2) a link labeled 'Begin short practice session'. The practice trials portion consisted of 3 keyword and risk web pages similar to those used in the 
experiment. The start the task page contained text that included: 1. Locate each of the keywords, 2. Complete a short demographics form and 3) a link label 'Ready to start the task' (A7).

Target keyword pages. The target keyword pages consisted of a target keyword and a standard blue underlined link label 'Next'. An example screenshot of a target keyword page is provided in Appendix A (A8). Table 4 presents the list of keywords used as targets for both tasks.

Table 1. List of keywords used as targets for both tasks
(1) hair loss
(7) nose bleeds
(13) vivid dreams
(2) diarrhea
(8) dizziness
(14) depression
(3) hearing loss
(9) liver disease
(15) pancreatic cancer
(4) heart burn
(10) nightmares
(16) tiredness
(5) indigestion
(11) incontinence
(17) impotence
(6) seizure
(12) nausea
(18) anxiety

Risk web pages. Two prescription drug risk information web pages were digitally captured from the Wellbutrin [depression] and Advair [asthma] manufacturers' web sites. Example screenshots for an Advair and Wellbutrin risk web page are provided in Appendix A (A8, A10, A11 and A12). The left and top portions of the web pages were preserved while only removing the center content portion on the pages to create two visual templates. The target always present task used these same templates; however, the target present/absent task required the addition of present and absent buttons so two more templates were created. 
A set of six paragraphs of risk information (three per drug) were put together using risk information that was captured from current DTC prescription drugs manufacturers' web sites including: (Singulair, Advair [asthma] and Wellbutrin, Celexa, Paxil [depression]. These six paragraphs were then used to create nine Wellbutrin and nine Advair risk web pages for the $3 \times 3$ repeated measures design. For the target present task these 18 risk web pages were shown twice in a randomized order for a total of 36 trials per participant. For the target present/absent the same stimuli were used except that they had to be doubled to include both target present and absent trials. So for this task each participant had a total of 72 trials with 36 pages that had a target present and the other 36 a target was absent.

Each web page used the same paragraph of text (risk information) for each of the three paragraphs with the only difference being that the sentence order was randomized between them. Each paragraph of text was presented in a 12 point sans-serif font. The completed web page images with standardized risk information were then saved as high resolution JPG images.

Demographics. Participant demographics were collected using a web form to automate the data collection process. Appendix B presents a screenshot of the demographics web form used for the experiment.

\section{Procedure}

Both visual search tasks used the same procedure with the only difference being in the type of response required. Target always present task required participants to find a 
keyword and respond by using a mouse to click on one of three image hotspots overlaid over each of the three paragraphs on a risk web page (Figure 2). The target present/absent task required participants to search for a keyword and respond by using a mouse to click on either a present or absent button to indicate the target presence on a risk web page (Figure 3).

First, participants where introduced to the study and presented with an informed consent form (Appendix C). They were then instructed to bring up Internet explorer on their PC and to type in the testing URL. Next they logged into the experiment's web site, where they were then presented with a series of instructional pages for the specific task to which they were assigned (Appendix A). The experimenter took each participant through the overview page (A1) giving them a brief introduction to the task. Each participant then was then allowed to read through 2 more pages of basic instructions that introduced to the task and further emphasized the importance of accuracy in locating the target keywords (A2 and A3). Participants proceeded through each page by clicking on a 'Next' link available on each of the pages.

Upon completion of the basic instructions participants were presented with the visual demonstration page that automatically started playing when the page was loaded. Participants watched the short demonstration and could either click a link to reload the page / restarted the demonstration or they could proceed by clicking 'Next'. The next page was an intermediary page that provided a link label so participants could start the set of 3 practice trials similar to the ones used in the data collection portion of the experiment. After completing the last practice trial, participants presented with a page that included a link to start the experiment. 
After verbally confirming they understood what to do, participants were allowed to start the experimental trials (data collection portion) on their own. Upon completion of the experiment participants were presented with the demographics questionnaire to fill out (Appendix B). Finally, participants were debriefed about the experiment and then they were excused.

\section{RESULTS}

Sixty individuals participated, ages 18 to $57, M=27.93, S D=10.51$. Thirty participants were undergraduate students from North Carolina State University, who were enrolled in the introductory psychology course participant pool, ages 18 to $42, M=21.1, S D$ $=5.85$. The remaining 30 participants were non-students, ages 23 to $57, M=35.31, S D=$ 9.24, recruited from the local community in Raleigh, NC. Tables 2 and 3 present a summary of age, gender and student status demographic data collected for each task.

Table 4 presents a summary of participants' responses to the demographics questionnaire surveying experience with computers and the Internet including: 1) How

experienced are you at using computers?, 2) How experienced are you at surfing the web?, 3) How experienced are you at shopping on the web?, and 4) How often do you surf the web? 
Table 2. Gender and race demographics of participants

\begin{tabular}{|c|c|c|c|c|}
\hline Gender & $\underline{\mathrm{n}}$ & $\%$ & Race & $\underline{\mathrm{n}}$ \\
\hline Male & 39 & 65 & Caucasian & 47 \\
\hline Female & 21 & 35 & Other & 13 \\
\hline
\end{tabular}

$\underline{\text { Target always present task }}$

Male

1757 Caucasian

$19 \quad 63$

Female

1343 Other

$11 \quad 37$

Target present/absent task

Male

Female

$\begin{array}{ccl}22 & 73 & \text { Caucasian } \\ 8 & 27 & \text { Other }\end{array}$

$28 \quad 93$

27

Table 3. Age demographics of participants

Age $\quad \underline{\mathrm{M}} \quad \underline{\mathrm{SD}}$

Both tasks

$27.93 \quad 10.51$

Target always present task $\quad 29.03 \quad 11.30$

Target present/absent task $\quad 26.93 \quad 9.77$

Student $(\mathrm{n}=30) \quad 21.10 \quad 5.85$

Non-student $(\mathrm{n}=30) \quad 35.31 \quad 9.24$

Table 4. Experience with computers and Internet demographics data

How experienced are you at using computers?

$\underline{\mathrm{M}} \quad \underline{\mathrm{SD}}$

$I=$ not all experience to $5=$ extremely experienced

$4.15 \quad .79$

How experienced are you at surfing the web?

$4.26 \quad .75$

$I=$ not all experience to $5=$ extremely experienced

How experienced are you at shopping on the web? $\quad 3.59 \quad 1.10$

$1=$ not all experience to $5=$ extremely experienced

How often do you surf the web?

$1=$ never surf the web, 2=few times a year, 3=few times a month, 4=few times a week,

$5=$ few times $a$ day 
Although the experiment was a 3x3x2 mixed design the analysis consisted of $3 \times 3$ repeated measures analysis to examine the effects of highlighting validity and placement of target keywords on response time and accuracy for each task separately. Tasks were examined separately due to potential differences inherent to the nature of each of the tasks. Each task's analyses began with a multivariate analysis of variance (MANOVA) examining all dependent (response time and accuracy) and independent variables (Highlighting validity and Placement) together. Next, ANOVA's were conducted examining significant main effects and interaction effects found in the MANOVA analysis with the a priori comparisons of stated hypotheses being performed using paired t tests. For post-hoc comparisons, Tukey's Honestly Significant Difference (HSD) test was used.

ANOVA summary tables for each set of analyses are presented in Appendix D. The next section deals with the target always present task exclusively. It is followed by another major section describing the results for the target present/absent task.

\section{Target Always Present Task}

The MANOVA including response time and accuracy had a significant main effect for both highlighting validity, Wilkes Lambda $=40.57, F(3,56), p<.0001$, and placement, Wilkes Lambda $=16.33, F(3,56), p<.0001$, but failed to yield a significant interaction effect for highlighting validity and placement, Wilkes Lambda $=0.97, F(3,52), p$ $>.05$. 
Response time. Table 5 presents the mean response times for each level of highlighting validity. The effect of highlighting validity on response time was significant, $F$ $(2,28)=76.20, p<.0001$. Targets in the valid highlighting conditions were found significantly faster compared to targets in the invalid highlighting, $t(29)=154.11, p<.0001$, and no highlighting conditions, $t(29)=72.47, p<.0001$. Targets in the no highlighting conditions and targets in the invalid highlighting conditions were not significantly different in terms of response time, $t(19)=0.16, p>.10$.

Table 5 also presents the mean response times for each level of placement. The effect of placement on response time was significant, $F(2,28)=27.12, p<.0001$. Targets placed in the top of a web page were found significantly faster compared to targets placed in the bottom of a web page, $t(29)=33.58, p<.0001$, but were not found significantly faster compared to targets placed in the middle of a web page, $t(29)=1.02, p>.05$. Also targets placed in the middle of a web page were found significantly faster compared to targets placed in the bottom of a web page, $t(29)=42.97, p<.0001$. The two-way ANOVA (highlighting validity $\mathrm{x}$ placement) on response time failed to yield a significant interaction effect, $F$ ( 4 , 26) $=0.58, p>.05$. Mean scores of response time (seconds) for highlighting validity and placement are presented in Figure 4. 
Table 5. Response time in seconds as a function of highlighting validity $x$ placement (Target always present task)

$\begin{array}{lcccc} & \begin{array}{c}\text { Valid } \\ \text { highlighting }\end{array} & \begin{array}{c}\text { No } \\ \text { highlighting }\end{array} & \begin{array}{c}\text { Invalid } \\ \text { highlighting }\end{array} & \text { Mean } \\ \text { Top } & 3.73 & 15.97 & 18.49 & 12.73 \\ \text { Middle } & 4.80 & 18.87 & 17.80 & 13.82 \\ \text { Bottom } & 9.95 & 25.09 & 22.02 & 19.02 \\ \text { Mean } & 6.16 & 19.97 & 19.43 & \end{array}$

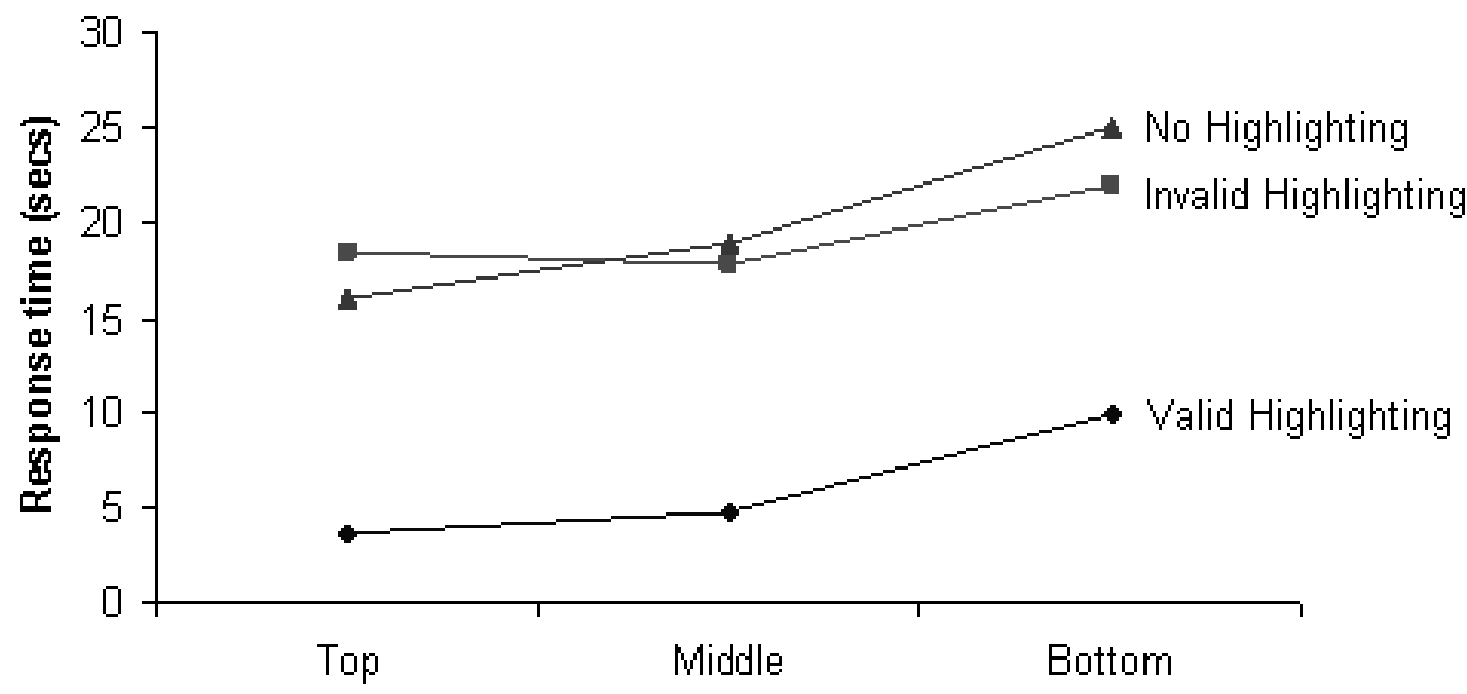

Figure 4. Response time as a function of highlighting validity x placement (Target always present task)

Accuracy. Table 6 presents the means for accuracy (proportion of correct responses) for each level of highlighting validity. The effect of highlighting validity on accuracy was significant, $F(2,28)=4.93, p<.05$. Targets in the valid highlighting conditions were found 
with significantly more accuracy compared to targets in the no highlighting conditions, $t$ (29) $=10.21, p<.01$, but with significantly less accuracy compared to targets in the invalid highlighting conditions, $t(29)=2.58, p>.05$. Targets in the no highlighting and invalid highlighting conditions were not significantly different in terms of accuracy, $t(29)=0.15, p$ $>.05$.

Table 6 also presents the means for accuracy for each level of placement. The effect of placement on accuracy was significant, $F(2,28)=5.53, p<.01$. Target keywords placed in the top of the web page were found with significantly better accuracy compared to the middle, $t(29)=4.68, p<.05$, and bottom of the web page, $t(29)=9.16, p<.01$. However, targets in the middle and bottom of the web page were not significantly different, $t(29)=$ $0.14, p>.05$. The two-way ANOVA (highlighting validity x placement) on accuracy failed to yield a significant interaction effect, $F(4,26)=1.36, p>.05$. Mean scores of accuracy (portion of correct responses) for highlighting validity and placement are also presented in Figure 5.

Table 6. Accuracy as a function of highlighting validity $x$ placement (Target always present task)

\begin{tabular}{lcccc} 
& $\begin{array}{c}\text { Valid } \\
\text { highlighting }\end{array}$ & $\begin{array}{c}\text { No } \\
\text { highlighting }\end{array}$ & $\begin{array}{c}\text { Invalid } \\
\text { highlighting }\end{array}$ & Mean \\
Top & 1.0 & .98 & .98 & .98 \\
Middle & 1.0 & .93 & .93 & .96 \\
Bottom & .98 & .93 & .95 & .95 \\
\hline Mean & .99 & .94 & .95 &
\end{tabular}




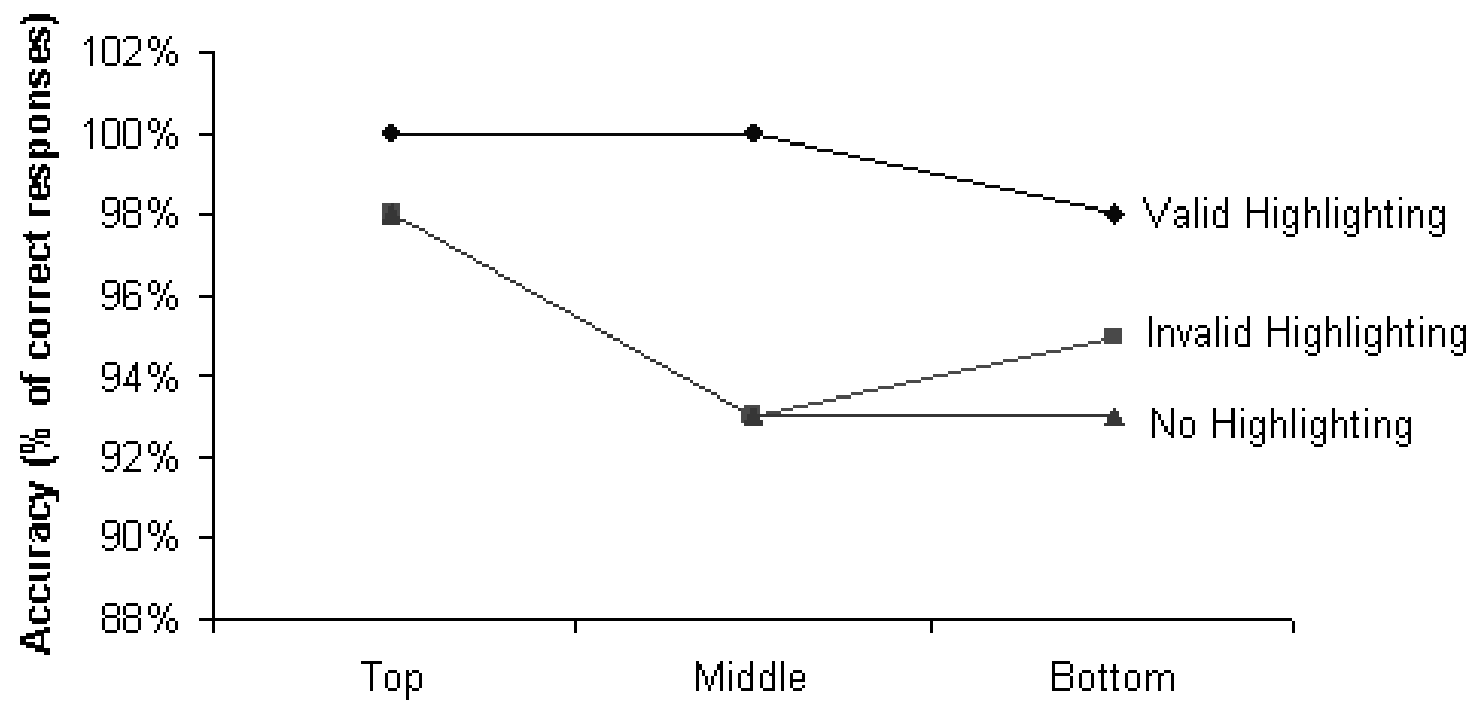

Figure 5. Accuracy as a function of highlighting validity $x$ placement (Target always present task)

\section{Target Present/Absent Task}

The MANOVA including response time and accuracy had a significant main effect for both highlighting validity, Wilkes Lambda $=81.85, F(3,56), p<.0001$, and placement, Wilkes Lambda $=16.99, F(3,56), p<.0001$, as well as a significant interaction effect, Wilkes Lambda $=9.42, F(3,52), p<.01$.

Response time. Table 7 presents the mean response times for each level of highlighting validity. The effect of highlighting on response time was significant, $F(2,28)$ $=135.61, p<.0001$. Targets in the valid highlighting conditions were found significantly faster compared to targets in the invalid highlighting conditions, $t(29)=206.05, p<.0001$, or no highlighting conditions, $t(29)=254.87, p<.0001$. Targets in the no highlighting 
conditions compared to targets in the invalid highlighting conditions were not significantly different in terms of response time, $t(29)=1.83, p>.05$.

Table 7 also presents the mean response times for each level of placement. The effect of placement on response time was also significant, $F(2,28)=23.87, p<.0001$. Targets placed at the top of a web page were found significantly faster compared to the targets placed at the bottom of a web page, $t(29)=36.35, p<.0001$, but not significantly faster compared to targets placed in the middle of a web page, $t(29)=1.70, p>.05$. Also targets were found significantly faster in the middle of a web page compared to the bottom of a web page, $t$ (29) $=35.52, p<.0001$.

Table 7. Response time in seconds as a function of highlighting validity $x$ placement (Target present/absent task)

\begin{tabular}{lcccr} 
& $\begin{array}{c}\text { Valid } \\
\text { highlighting }\end{array}$ & $\begin{array}{c}\text { No } \\
\text { highlighting }\end{array}$ & $\begin{array}{c}\text { Invalid } \\
\text { highlighting }\end{array}$ & Mean \\
Top & 2.91 & 11.04 & 12.28 & 8.75 \\
Middle & 3.13 & 13.27 & 11.96 & 9.45 \\
Bottom & 6.45 & 13.98 & 16.07 & 12.17 \\
\cline { 1 - 4 } Mean & 4.16 & 12.76 & 13.44 &
\end{tabular}

Table 8 presents the mean response times for each level of highlighting validity across all three placements. The effect of highlighting validity $\mathrm{x}$ placement interaction on response time was significant, $F(4,26)=2.80, p<.05$. Means for response time as a function of highlighting validity and placement are presented in Figure 6. Targets in the valid highlighting conditions were found significantly faster than all other targets in the invalid and 
no highlighting conditions across all three placements. Also targets in the invalid and no highlighting conditions were not significantly different across all three placements. Although not significant the pattern of means showed a trend that targets in the no highlighting conditions placed at the top and bottom of a web page were found faster than targets in the invalid highlighting condition in the same placements. However, targets in the invalid highlighting conditions placed in the middle of a web page were found faster than targets in the no highlighting conditions placed in the middle of a web page.

Table 8. Response time in seconds as a function of highlighting validity x placement (Target present/absent task)

\begin{tabular}{|c|c|}
\hline Valid \& Top & 2.91 \\
\hline Valid \& Middle & 3.13 \\
\hline Valid \& Bottom & 6.45 \\
\hline Invalid \& Top & 12.28 \\
\hline Invalid \& Middle & 11.96 \\
\hline Invalid \& Bottom & 16.07 \\
\hline No highlighting \& Top & 11.04 \\
\hline No highlighting \& Middle & 13.27 \\
\hline No highlighting \& Bottom & 13.98 \\
\hline
\end{tabular}




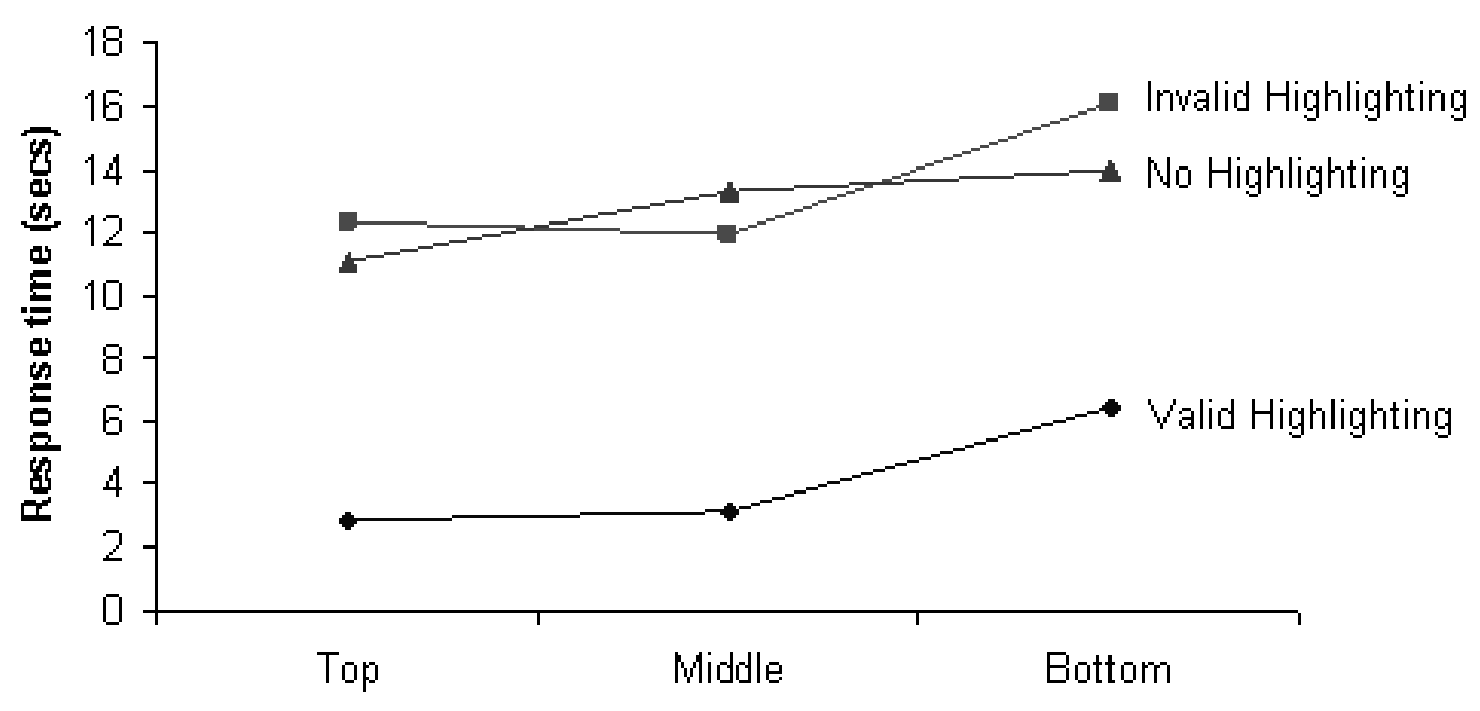

Figure 6. Mean response times as a function of highlighting validity x placement (Target present/absent task)

Accuracy. Table 9 presents the means for accuracy for each level of highlighting validity. The effect of highlighting validity on accuracy was significant, $F(2,28)=30.15, p$ $<.0001$. Targets with valid highlighting were found with significantly more accuracy compared to targets in the invalid highlighting conditions, $t(29)=72.80, p<.0001$, or no highlighting conditions, $t(29)=52.46, p>.0001$. Comparing targets in the no highlighting conditions to targets in the invalid highlighting conditions there was not a significant difference, $t(29)=1.28, p>.05$.

Table 9 also presents the means for accuracy for each level of placement. The effect of placement on accuracy was also significant, $F(2,28)=8.46, p<.001$. However, targets placed at the top of a web page were found to be significantly less accurate compared to the middle, $t(29)=16.0, p<.001$, and the bottom of a web page, $t(29)=10.75, p<.01$. Targets 
in place in the middle of a web page were not significantly different compared to the bottom of a web page, $t(29)=1.28, p>.05$.

Table 9. Accuracy as a function of Highlighting validity $x$ Placement (Target present/absent task)

\begin{tabular}{lcccc} 
& $\begin{array}{c}\text { Valid } \\
\text { highlighting }\end{array}$ & $\begin{array}{c}\text { No } \\
\text { highlighting }\end{array}$ & $\begin{array}{c}\text { Invalid } \\
\text { highlighting }\end{array}$ & Mean \\
Top & .99 & .63 & .53 & .72 \\
Middle & .98 & .77 & .74 & .83 \\
Bottom & .98 & .73 & .76 & .82 \\
\hline Mean & .98 & .71 & .68 &
\end{tabular}

The effect of highlighting validity and placement was significant, $F(4,26)=7.03, p$ $<.001$. Mean scores of accuracy (portion of correct responses) for highlighting validity and placement are presented in Table 9 and Figure 7. Targets with valid highlighting in all three placements were found significantly more accurately than targets with invalid and no highlighting at any placement. Also targets in the invalid highlighting conditions placed at the top of web page were significantly the worst in terms of accuracy compared to all other combinations of highlighting and placement. Table 10 presents the means for accuracy for each level of highlighting validity. Although not significant the pattern of means showed a trend that targets in the no highlighting conditions placed in the top and middle of a web page were found with greater accuracy than targets in the invalid highlighting conditions in the same positions. However, when targets in the invalid highlighting conditions were placed at the bottom of a web page they were found with greater accuracy than targets in the no highlighting conditions also placed at the bottom. 
Table 10. Accuracy as a function of highlighting validity $x$ placement (Target present/absent task)

Valid \& Top

Valid \& Middle

Valid \& Bottom

Invalid \& Top

Invalid \& Middle

Invalid \& Bottom

No highlighting \& Top

No highlighting \& Middle

No highlighting \& Bottom
Mean

.99

.98

.98

.53

.74

.76

.63

.77

.73
Tukey

A

A

A

C

B

B

B

B

B

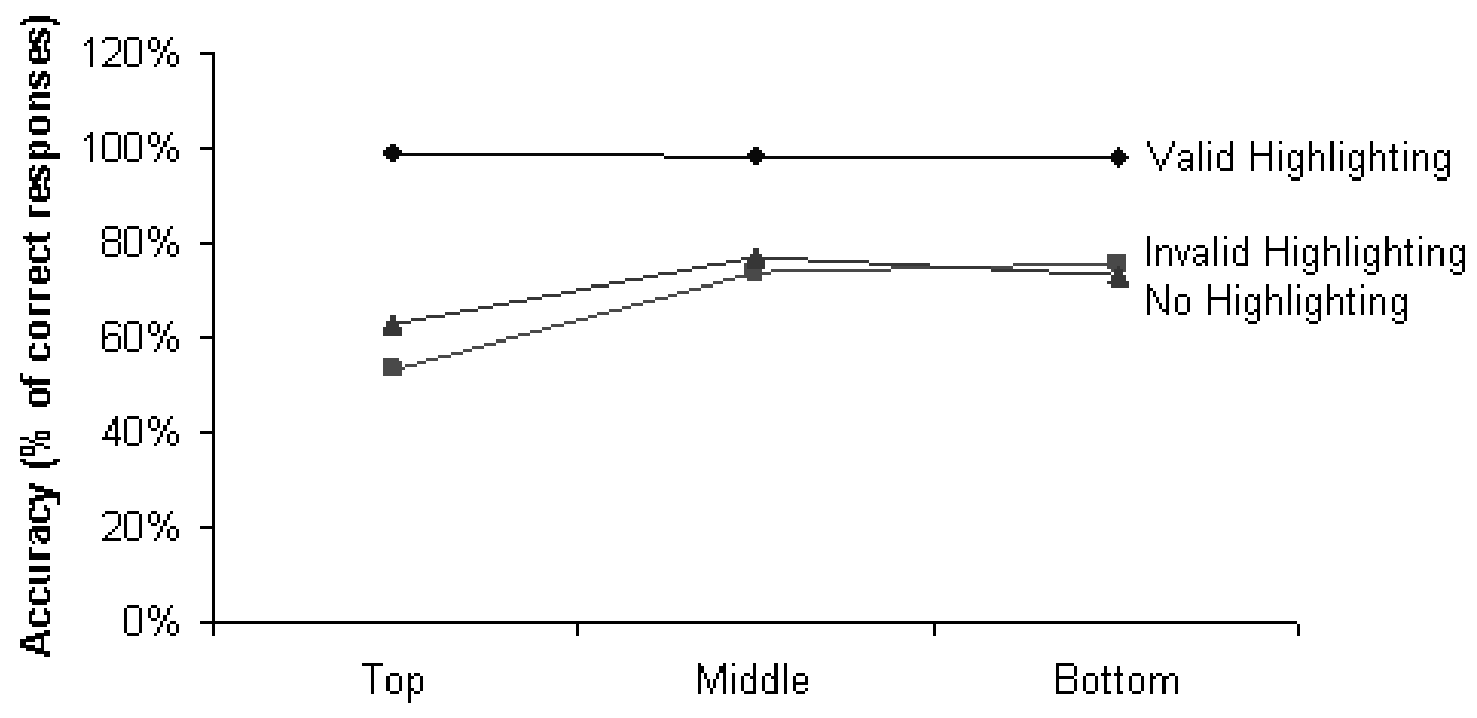

Figure 7. Accuracy as a function of highlighting validity x placement (Target present/absent task) 


\section{DISCUSSION}

The purpose of the present study was to determine the effect of highlighting validity and placement of target keywords on response time and accuracy within DTC web pages. The results indicate that valid highlighting reduced response time and increased accuracy across both tasks. Also for both tasks there was no significant difference between invalid and no highlighting on response time or accuracy. Placement had a significant effect for both tasks with the results indicating that placement above the fold on a web page (Top and Middle) reduced response time and increased accuracy compared to placement at the bottom. Results showed no interaction effect for the target always present task but there was a significant interaction effect for the target present/absent task on both response time and accuracy.

Specific results are discussed in the following sections. Next, implications are addressed with particular emphasis placed on potential real world application. Finally, limitations of the present study are discussed, as well as suggestions for future research on the use of colored highlighting.

\section{Highlighting Validity}

For both tasks, target keywords in the valid highlighting conditions were detected significantly faster and with more accuracy than target keywords in the invalid or no highlighting conditions (Hypothesis 1 and 2). However, there was not a significant difference 
between the invalid highlighting and no highlighting conditions on response time (Hypothesis 3) and accuracy.

These results suggest that color highlighted targets will be found faster and with greater accuracy than in web pages containing no highlighting. This is consistent with previous research showing that stimuli differing in color from a homogeneous background can be detected easily by observers (Treisman \& Gelade, 1980). Furthermore, Tan and Fisher (1989) found color highlighting had significantly better performance than a non-highlighted condition. These results further indicate that color highlighting provided a benefit in response time and accuracy without any significant cost in performance across both tasks. These findings give further support to the “color advantage effect” identified in Martin et al.'s (1987) research that suggested that the use of colored highlighting may be an optimal form of highlighting compared to other forms. Additional details on the potential uses of colored highlighting are discussed in the Implications section

\section{Placement}

Results also indicate that keyword targets located above the fold of a web page (Top and Middle) were found quicker than targets placed below the fold (Bottom) in both visual search tasks (Hypothesis 4). Specifically, target keywords located in the top and middle paragraphs were found significantly faster than targets placed at the bottom of the page. This result was most likely being due to the requirement of participants having to scroll to see the target located in the bottom paragraph as well as scanning a web page from the top down. 
Target keywords placed above the fold were located with more accuracy than below the fold in the Target always present task only. Interestingly, results of the target present/absent task indicated that the placement in the top paragraph can negatively affect the accuracy of locating a target keyword. This result may be more of a function of the interaction of task and placement versus just placement alone. This could be based on the influence a participant's expectancy of target being present could have on accuracy. Also a participant's visual scan pattern could have an effect because a participant may miss the target keyword in the top paragraph if they start scanning at the top and go down the page. This could potentially decrease the likelihood of locating the target keyword if it was missed in the first visual pass.

Overall, these results suggest that targets placed above the fold will be found faster and with greater accuracy than when placed below the fold. These findings provide some quantitative support for the web design guideline / convention of placing important information above the fold (Nielsen, 2000; Price \& Price, 2002; Spool et al., 1999). Also research with DTC web pages has shown that risk information is less likely to be found if scrolling is required (Vigilante and Wogalter, 2005). Recommendations and guidelines for placement of risk information are further discussed in the Implications section below.

\section{Highlighting validity and Placement}

The results indicate that valid highlighting reduced both response time and increased accuracy for both tasks. Also there was no significant difference between invalid and no 
highlighting conditions on response time or accuracy for both tasks. The results also indicated that placement above the fold on a web page (Top and Middle) significantly reduced response time and increased accuracy compared to placement at the bottom for both tasks.

Results showed no interaction effect for the target always present task but there was a significant interaction effect for the target present/absent task on both response time and accuracy. Targets in the valid highlighting conditions across all 3 placements were located significantly faster and with more accuracy than targets presented in all three levels of highlighting validity across all 3 placements. This result suggests that valid highlighting is optimal in terms of performance compared to invalid and no highlighting. Results further indicated that valid highlighting in any placement on a web page was superior compared to all other levels of highlighting validity.

An interesting trend in the results showed that targets in the no highlighting conditions placed in the middle of a web page were found faster than targets in the invalid highlighting conditions placed in the middle of a web page. However, targets in the invalid highlighting conditions placed at the top and bottom of a web page were found faster than targets in the no highlighting conditions with the same placements. This finding may be in part due to participants learning that if a paragraph had a distracter keyword highlighted that a target was not going to be in the same paragraph. This could then prompt them to start scanning the remaining two paragraphs and thereby potentially reducing their response time. 
Targets in the invalid highlighting conditions placed at the top of web page were found with significantly less accuracy than all other targets across all three placements. However, when targets in the invalid highlighting conditions were placed at the bottom they were found faster than targets in the no highlighting conditions also placed at the bottom. This result suggests that the placement at the top of a web page may not always be ideal when a distracter is highlighted (Invalid highlighting) because it produced more errors.

Overall the analyses for both tasks showed that valid highlighting had a benefit and that invalid highlighting had no cost compared to no highlighting with this small exception. Since this finding was task dependent it suggests that it is something in the target present/absent task itself. This may in part be attributed to the condition that participants' had to complete twice the number of trials compared to the target always present task where this pattern was not indicated. Potentially due to the increased number of trials participants may have experienced some fatigue when doing a repetitive visual scanning task across a number of pages with a large amount of text. Finally, this particular result requires further investigation and needs to be replicated in future research to determine if can be replicated and there by shown to a real effect.

\section{Implications}

Results for the current study suggest that valid highlighting and placement above the fold (Top and Middle) of a web page could help facilitate consumers looking for information. The use of colored highlighting on the web help provide consumers a way to visually filter 
extraneous information at the page level. Colored highlighting could be used in many of web driven applications such as highlighting different types of risk information available in prescription drug manufacturers' DTC web pages or even in something like a prescription drug insert or a material safety data sheet available in PDF and displayed in a browser. Even with these examples there is still the central issue of making highlighting valid to a user. The question of validity can be addressed through either: 1) personalized color highlighting of information to an individual or 2) customized highlighting to a particular information domain.

Personalized colored highlighting might be based on individual interests or particular safety concerns which are made salient versus other extraneous information. Collecting and utilizing this type of information for a given individual could serve as a way to make the information desired validly highlighting. Also by tailoring the highlighting to a specific user could greatly reduce the potential for invalid highlighting. Letting the user choose what information they want highlighted is one way to increase validity but the risk is that they may miss something important in the text.

Another way to potentially increase highlighting validity for a user is to customize it to a particular information domain versus what is important to a given individual. In this example, keywords associated to a domain, like prescription drug information, could be what would be highlighted in a user's browser. This implementation could automatically highlight all relative risk information such as side effects and interactions whenever a user visits any manufacturers' prescription drug web site. Customized highlighting could be more beneficial 
compared to personalized highlighting because it would allow for all related risk information to be highlighted not just what is important to an individual. The only tradeoff with this approach is that it could increase the potential for invalid highlighting. Overall, highlighting showed benefits when the sought information is validly highlighted but has no cost when different information is highlighted. The present findings also confirm previous results found other studies done in different domains.

Finally, results also indicated the importance of placement on a web page. There is a need for the FDA to provide updated guidelines for placement of risk information on manufacturers' DTC web sites. The current guidelines for the web were written to regulate print and broadcast media so they are not conducive to presentation of information on the Internet. Placing important information such as risks and side effects above the fold on web pages could increase the overall likelihood that a consumer can find it. This finding further supports our need for the FDA to establish more specific design guidelines for presenting risk information on prescription drug manufacturers' DTC web pages.

\section{Limitations and Future Research}

The present study sought to examine the effects of the validity and placement of colored highlighted keywords on DTC manufacturers' web pages. However, this study only examined the effects of validity highlighting and placement with single target keywords versus having a highlighted target surrounded by multiple highlighted distracters on a web page. Various numbers of distracters could be highlighted to examine the effect of increasing 
the amount invalid. Also future research could look at the use of color coded highlighting of different types of risk information (e.g., side effects, adverse effects, interactions). This could potentially extend the use of highlighting by coding different types of information to a particular color. One example would be to highlight interactions in yellow and side effects in red.

In addition to examining the use of highlighting on individual web pages, research should also examine it within web sites with multiple pages and internal navigation. Web sites are therefore much more complex than web pages and require further examination. With this in mind, other methods could be researched to further facilitate finding risk information on manufacturers' DTC prescription drug web pages. 


\section{REFERENCES}

Aiken, K. J. (2002). Direct-to-consumer advertising of prescription drugs: Patient survey results. January 18, 2004, http://www.fda.gov/cder/ddmac/Presentations/KitHMCC2002out/

Barlow, T. and Wogalter, M. S. (1993). Alcoholic beverage warnings in magazine and television advertisements. Journal of Consumer Research, 20, 147-156.

Bell, R. A., Kravitz, R. L., and Wilkes, M. S. (1999). Direct-to-consumer prescription drug advertising and the public. Journal of General Internal Medicine, 14, 651-657.

Calfee, J. E. (2002). Public policy issues in direct-to-consumer advertising of prescription drugs. Journal of Public Policy \& Marketing, 21, 174-193.

Everett, S. E. (1991). Lay audience response to prescription drug advertising. Journal of Advertising Research, 31, 43-49.

Food and Drug Administration [FDA]. (1999). Attitudes and behaviors associated with direct-to-consumer (DTC) promotion of prescription drugs: Preliminary survey results. Washington, DC: Office of Medical Policy, Division of Drug Marketing, Advertising, and Communication. Retrieved January 18, 2004, from http://www.fda.gov/cder/ddmac/DTCtitle.htm

Food and Drug Administration. (1999a). Prescription-drug advertisements (Federal Register, Title 21 Vol. 4, Parts 200-299). Washington, DC: U.S. Government Printing Office. 
Food and Drug Administration. (1997). Draft guidelines for industry: Consumer-directed broadcast advertisements. Federal Register, 62(155), 43171-43193.

Food and Drug Administration. (1999). Attitudes and behaviors associated with direct-toconsumer (DTC) promotion of prescription drugs: Preliminary survey results. Washington, DC: Office of Medical Policy, Division of Drug Marketing, Advertising, and Communication. Retrieved December 28, 2002, from http://www.fda.gov/cder/ddmac/DTCtitle.htm

Food and Drug Administration. (1999). Guidance for industry: Consumer-directed broadcast advertisements. Retrieve January 22, 2004, from http://www.fda.gov/cder/guidance/1804fnl.pdf

Frantz, J. P. and Rhoades, T. P. (1993). A task-analytic approach to the temporal and spatial placement of product warnings. Human Factors, 35, 719-730.

Hicks, K. E., Wogalter, M. S., and Vigilante, W. J. (2005). Placement of Benefits and Risks in Prescription Drug Manufacturers' Web sites and Information Source Expectations. Drug Information Journal, 39, 267-278.

Hink-Eustace, J .K. (2000). Highlighting cues in visual search: the color advantage effect, Unpublished master's thesis, North Carolina State University, Raleigh, NC.

Jonides, J. and Yantis, S. (1988). Uniqueness of abrupt stimulus onset in capturing attention. Perception \& Psychophysics, 43, 346-354. 
Kalsher, M. J. and Wogalter, M. S. (2006). Influence of presentation modality on communication of pharmaceutical risk information in direct-to-consumer (DTC) television commercials. In R. N. Pikkar, E. A. P. Koningsveld, and P. J. M. Settels (Eds.), Proceedings of the XVIth Triennial International Ergonomics Association (pp. 5149-5154). Amsterdam: Elsevier (ISSN 0003-6870).

Lynch, P. J. and Horton, S. (1999). Web style guide: Basic design for creating web sites. New Haven, CT: Yale University Press. http://info.med.yale.edu/caim/manual/ Martin, D. W., McDonald, D. R., and Patton, C. R., (1987). The Benefit/Cost of VDT Highlighting. In G. Salvendy, S.L. Sauter and J.J. Hurrell., (Eds.), Advances in Human Factors / Ergonomics, 10A: Social, Ergonomic and Stress Aspects of Work with Computers (pp. 89-96). Amsterdam: Elsevier Science Publishers B.V.

Matlin, M. W. (2002). Cognition. Orlando, Florida: Harcourt, Brace, Jovanovich.

Nielsen, J. (2000). Designing web usability: The practice of simplicity, Indianapolis: New Riders.

Price, J. and Price, L. (2002). Hot text: Web writing that works, Indianapolis: New Riders. Spool, J. M., Scanlon, T., Schroeder, W., Snyder, C., and DeAngelo, T. (1999). Web site usability: a designer's guide, San Francisco: Morgan Kaufmann Publishers.

Strawbridge, J. A. (1986). The influence of placement, highlighting, and imbedding on warning effectiveness. Proceedings of the Human Factors Society and Ergonomics 30th Annual Meeting (pp. 716-720). Santa Monica, CA: Human Factors Society. 
Tan, K.C., and Fisher, D.L. (1989) Visual Displays: The Highlighting Paradox . Human Factor, 31, 17-30.

Treisman, A. M. and Gelade, G. (1980). A feature-integration theory of attention. Cognitive Psychology, 12, 97-136.

Vigilante, W. J. and Wogalter, M. S. (2005), Assessing Risk and Benefit Communication in Direct-to-Consumer Medication Web site Advertising. Drug Information Journal, 39, 3-12.

Wickens, C. D. and Hollands, J. G. (2000). Engineering psychology and human performance $\left(3^{r d} e d\right)$. Upper Saddle River, New Jersey: Prentice Hall.

Wilkes, M. S., Bell, R. A., and Kravitz, R. L. (2000). Direct-to-consumer prescription drug advertising: trends, impact, and implications. Health Affairs, 19, 110-128.

Wogalter, M. S. (2006). Communication-human information processing (C-HIP) model (Chap. 5). In M. S. Wogalter (Ed.) Handbook of Warnings (pp. 51-61). Mahwah, NJ: Lawrence Erlbaum Associates.

Wogalter, M. S., Paine, C. S., Mills, B. J., and Smith-Jackson, T. L. (1999). Application of cognitive principles to the design of direct-to-consumer advertising of prescription medications. In Proceedings of the Human Factors and Ergonomics Society 43rd Annual Meeting (pp. 515-519). Santa Monica, CA: The Human Factors and Ergonomics Society.

Wogalter, M. S., Godfrey, S. S., Fontenelle, G. A., Desaulniers, D. R., Rothstein, P. R., and Laughery, K. R. (1987). Effectiveness of warnings. Human Factors, 29, 599-612. 
Wogalter, M. S., Kalsher, M. J., and Racicot, B. M. (1993). Behavioral compliance with warnings: Effects of voice, context, and location. Safety Science, 16, 637-654.

Wogalter, M. S. and Leonard, S. D. (1999). Attention capture and maintenance. In M. S. Wogalter, D. M. DeJoy, \& K. R. Laughery (Eds.), Warnings and Risk Communication (pp. 123-148). London: Taylor and Francis.

Yantis, S. and Jonides, J. (1990). Abrupt visual onsets and selective attention: Voluntary vs. automatic allocation. Journal of Experimental Psychology: Human Perception and Performance, 16, 121-134.

Yantis, S. and Jonides, J. (1984). Abrupt visual onsets and selective attention: Evidence from visual search. Journal of Experimental Psychology: Human Perception \& Performance, 10, 601-621.

Young, S. L. and Wogalter, M. S. (1990). Comprehension and memory of instruction manual warnings: Conspicuous print and pictorial icons. Human Factors, 32, 637-649. 
APPENDICES 


\section{APPENDIX A}

\section{Example Screenshots}

\section{A1: Overview of experiment page}

\section{Overview}

1. Go thru some basic instructions

2. View a short demonstration of the task

3. Get some practice, before starting

4. Start the task $(20-25$ mins $)$

\section{$\underline{\text { Next }}$}

\section{A2: Basic instructions page one}

\section{Basic Instructions}

The basic task is to locate a keyword on a web page.

Please concentrate on locating the keyword correctly, please do not hurry

Next

\section{A3: Basic instructions page two}

\section{Basic Instructions}

The mouse is the only thing you need to use for the entire task

MMPORTANT: Please do not use the back button. 
A4: Demonstration of task page (Target always present task)

\section{Demonstration of task}

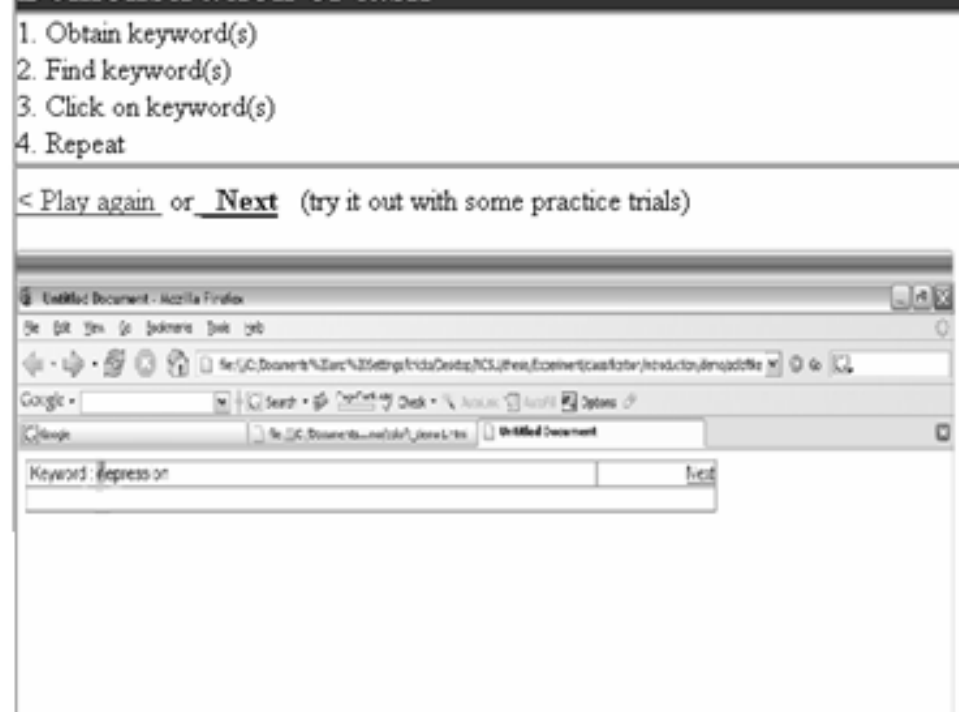

A5: Demonstration of task page (Target present/absent task)

\section{Demonstration of task}

1. Obtain keyword

2. look thru web page

3. click on Present or Absent

4. Repeat

$<$ Play again or Next (try it out with some practice trials)

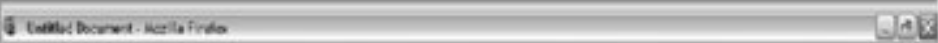

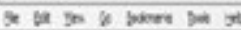

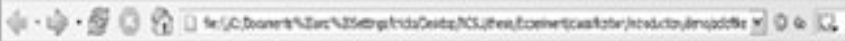

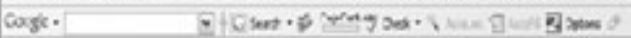

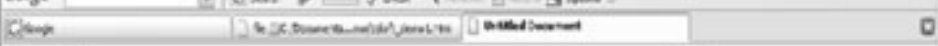

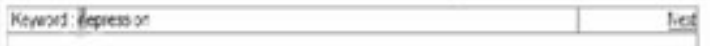


A6: Get some practice before starting page

Get some practice before starting

Try it out before beginning the experiment.

Begin short practice session

A7: Start the task page

\section{Start the task}

1. Locate each of the keywords.

2. Complete a short demographics form.

Ready to start the task

A8: Target keyword page

Keyword: Incontinence 
A9: Target always present task - Risk page (Wellbutrin)

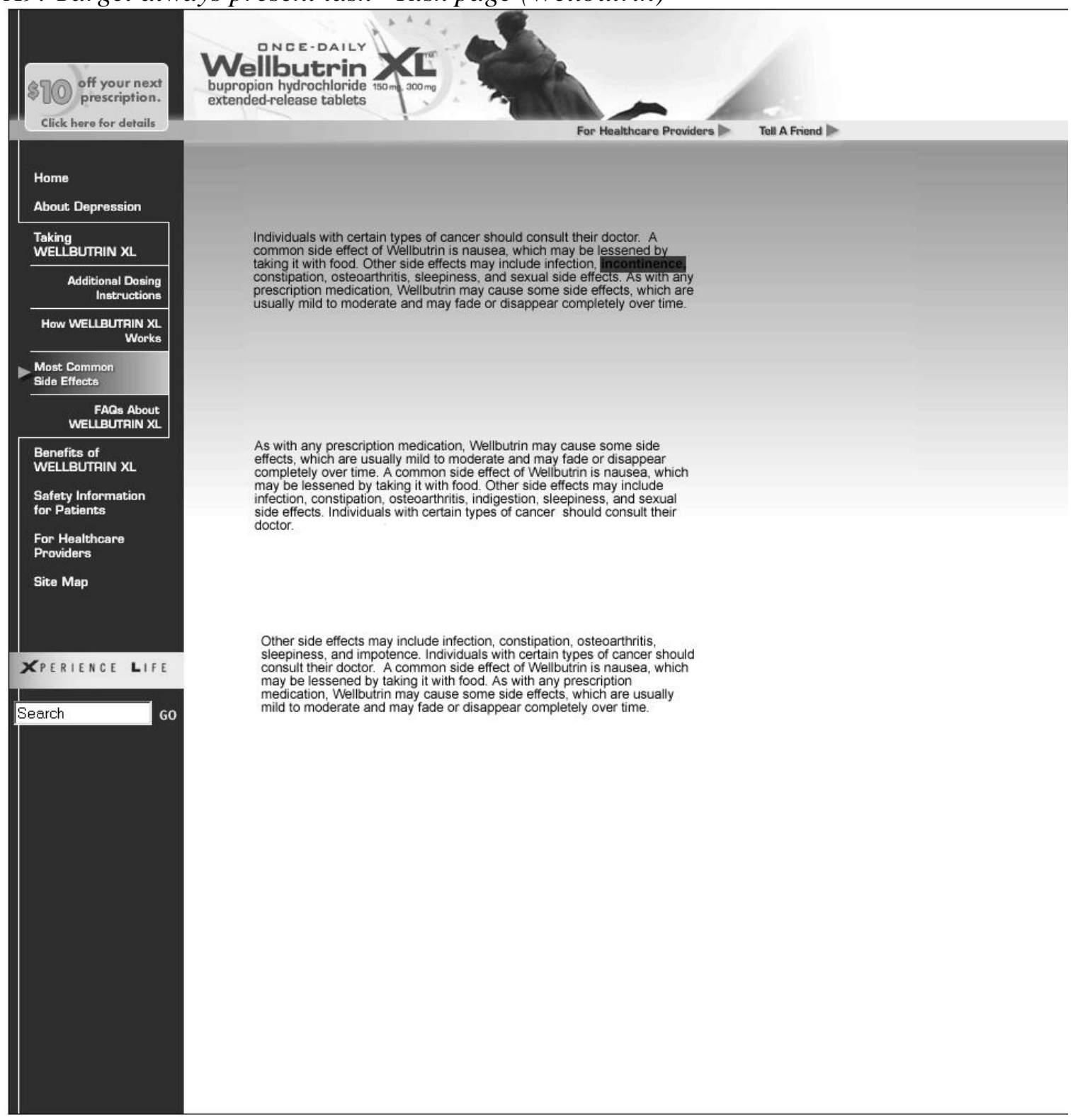




\section{A10: Target always present task - Risk page (Advair) \\ ADVAIR DISWUS 100/5 \\ Glossary | Site map | Important information about ADVAIR

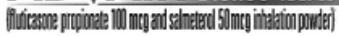 \\ Search: \\ $\mapsto G \circ$}

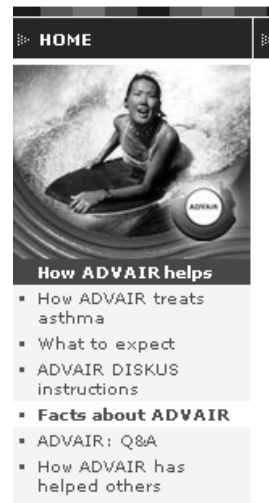

\begin{tabular}{|l|l|}
\hline HOW HDVAIR HELPS & WHAT IS ASTHMA? \\
\hline
\end{tabular}

TREATING ASTHMA

LIVING WITH ASTHMA

Facts about ADVAIR DISKUS ${ }^{\circledR}$

For patients 4 to 11 years old, ADVAIR 100/50 is for those who have asthma symptoms while on an inhaled corticosteroidcontrol. For asthma, ADVAIR should be taken once a day, in the evening as prescribed, whether or not you have asthma symptoms. Tell your doctor if you have heart condition or high blood pressure. The most riequent side ellects reported with ADVAIR are nausea, depression problems with ejaculation,
somnolence, and anorgasmia.

For asthma, ADVAIR should be taken once a day, in the evening as prescribed, whether or not you have asthma symptoms. Tell your doctor if you have a heart condition or high blood pressure. Th. Tost frequent side effecls ADVAIR $100 / 50$ is for those who have asthme symptoms while on an inhaled corticosteroidcontrol.

The most frequent side effects reported with ADVAIR are nausea, diarrhea problems with ejaculation, somnolence, and anorgasmia. Tell your doctor you have a heart condition or high blood pressure. For asthma, ADVAIR should be taken once a day, in the evening as prescribed, whether or not you have asthma symploms. For pallents 4 s pre 11 years old, ADVAIR 100/50

corticosteroidcontrol. 


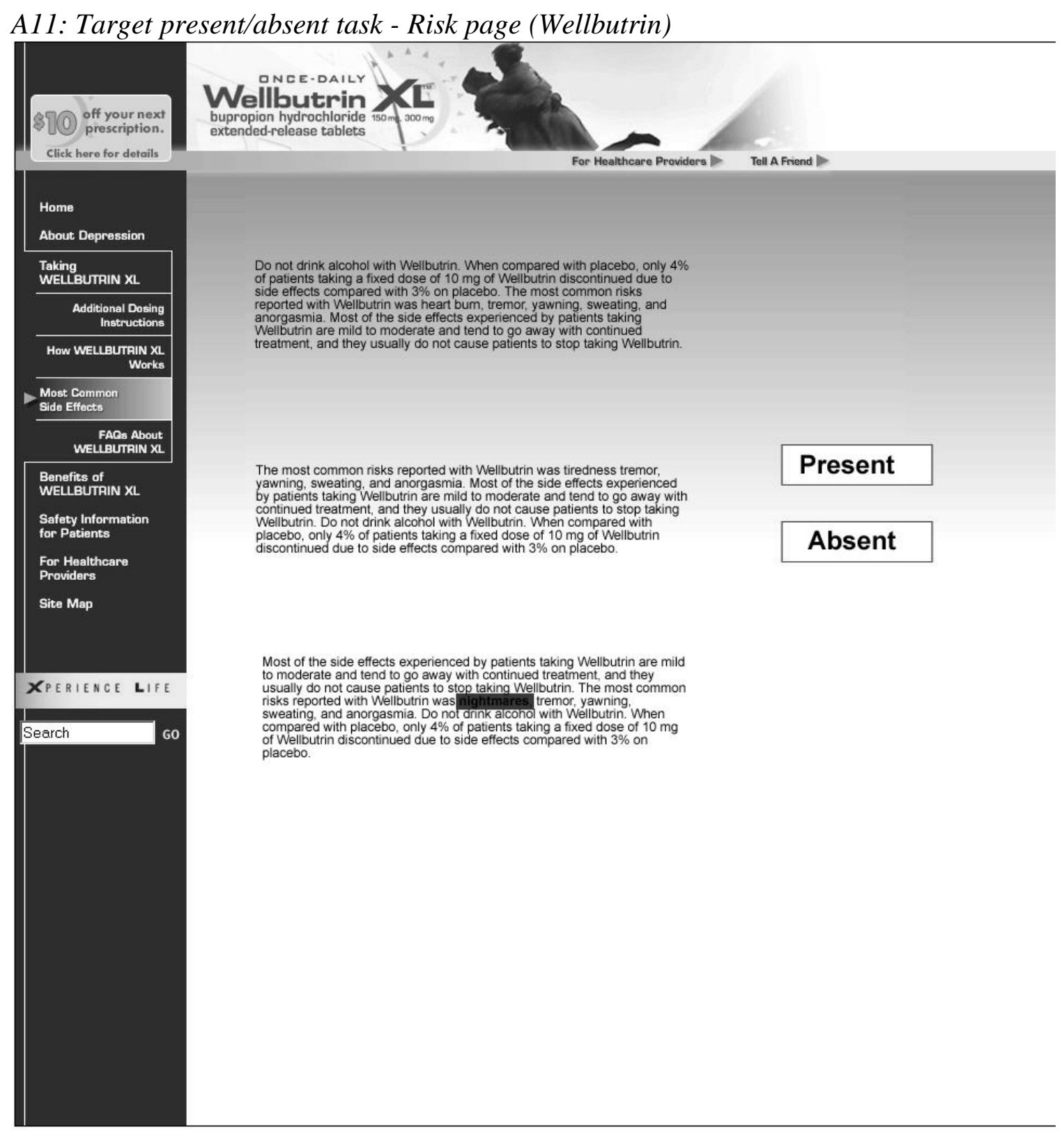


A12: Target present/absent task - Risk page (Advair)

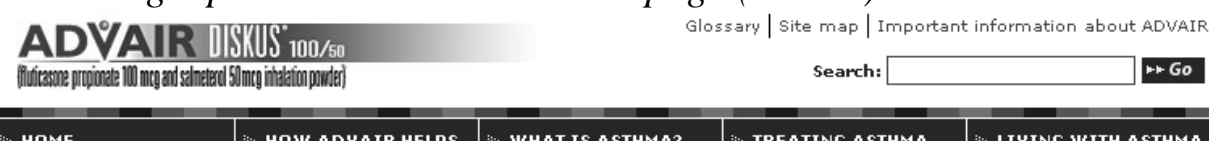

\begin{tabular}{|l|l|l|l|l|}
\hline HOME & HOW ADVAIR HELPS & WHAT IS ASTHMA? & TREATING ASTHMA & LIVING WITH ASTHMA \\
\hline
\end{tabular}

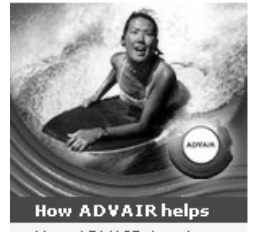

Facts about ADVAIR DISKUS ${ }^{\circledR}$

For patients 4 to 11 years old, ADVAIR 100/50 is for those who have asthma symptoms while on an inhaled corticosteroidcontrol. For asthma, ADVAIR should be taken once a day, in the evening as prescribed,
whether or not you have asthma symptoms. Tell your doctor if you have a How ADVAIR treats heart condition or high blood pressure. The most frequent side effects

asthma reported with ADVAIR are nausea
somnolence, and anorgasmia

- advair diskus

instruction

Facts about ADYAIR

- advair: Q\&a

How ADVAIR has
helped others

For asthma, ADVAIR should be taken once a day, in the evening as prescribed, wherter or not you have asthma symptoms. Tell your doctor if you have a heart condition or high blood pressure. The most frequent side ejaculation, somnolence, and anorgasmia. For patients 4 to 11 years old
ADVAIR $100 / 50$ is for those who have asthma symptoms while on an inhaled corticosteroidcontrol.

Present

Absent

The most frequent side effects reported with ADVAIR are nausea, diarrhea, problems with ejaculation, somnolence, and anorgasmia. Tell your doctor you have a heart condition or high blood pressure. For asthma, ADVAIR you have asthma symptoms. For patients 4 to 11 years old ADVAIR $100 / 50$ is for those who have asthma symptoms while on an inhaled corticosteroidcontrol. 


\section{APPENDIX B}

\section{Demographics Questionnaire}

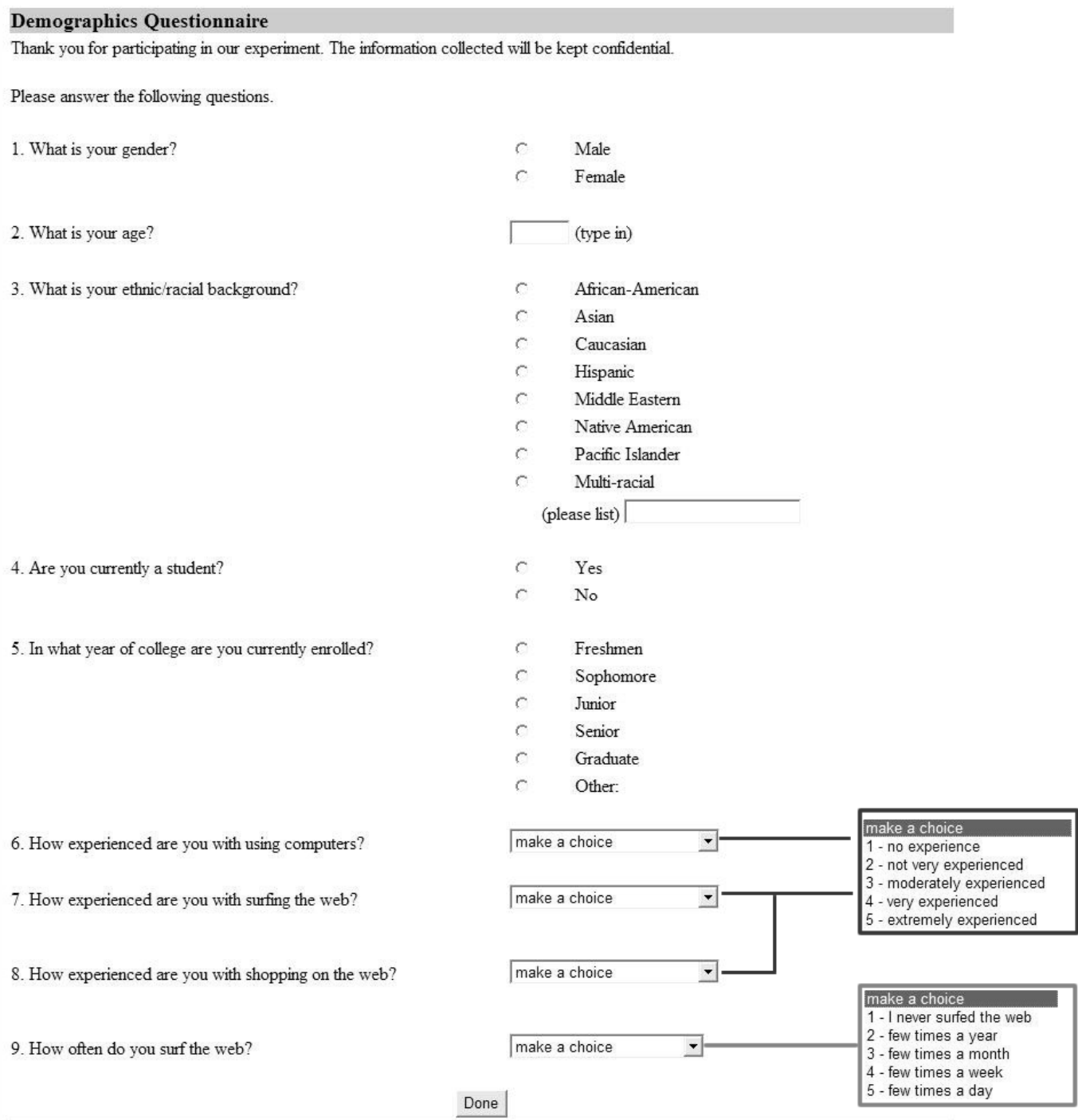




\title{
APPENDIX C
}

\section{Informed Consent Form}

\author{
North Carolina State University - INFORMED CONSENT FORM \\ Title of Study: Evaluating the use of highlighting safety information in web pages \\ Principle Investigator: Kevin Hicks
}

You are invited to participate in a research study. The purpose of this study is to evaluate exerpts from several primetime news programs to determine their importance and appeal.

INFORMATION

The study should take less than $\mathbf{4 0}$ mins to complete.

RISKS

There are no known risks associated with this study.

BENEFITS

The only direct benefit the participant can expect to receive is experimental credit.

CONFIDENTIALITY

The information in the study records will be kept strictly confidential. Data will be stored securely and will be made available only to persons conducting the study unless you specifically give permission in writing to do otherwise. No reference will be made in oral or written reports that could link you to the study.

\section{COMPENSATION}

For participating in this study you will receive $\mathbf{2}$ credits. If you withdraw from the study prior to its completion, you will receive 1 credit.

\section{CONTACT}

If you have questions at any time about the study or the procedures, you may contact the researcher, Kevin Hicks, at POE 740, or 515-8260. If you feel you have not been treated according to the descriptions in this form, or your rights as a participant in research have been violated during the course of this project, you may contact Dr. Matthew Zingraff, Chair of the NCSU IRB for the Use of Human Subjects in Research Committee, Box 7514, NCSU Campus (919/513-1834) or Mr. Matthew Ronning, Assistant Vice Chancellor, Research Administration, Box 7514, NCSU Campus (919/513-2148)

\section{PARTICIPATION}

Your participation in this study is voluntary; you may decline to participate without penalty. If you decide to participate, you may withdraw from the study at any time without penalty and without loss of benefits to which you are otherwise entitled. If you withdraw from the study before data collection is completed your data will be returned to you or destroyed.

\section{CONSENT}

I have read and understand the above information. I have received a copy of this form. I agree to participate in this study.

Participant's signature

Date

Print Name

Investigator's signature Date 


\section{APPENDIX D}

\section{ANOVA Summary Tables}

ANOVA Summary Table 1

Response Time. Target always present task x Highlighting validity (Valid, Invalid \& No) x Placement (Top, Middle \& Bottom) repeated measures ANOVA.

\begin{tabular}{lrrrccc} 
Source of Variation & df & \multicolumn{1}{c}{$\begin{array}{c}\text { Sum of } \\
\text { Squares }\end{array}$} & $\begin{array}{c}\text { Mean } \\
\text { Square }\end{array}$ & F & p & $\begin{array}{c}\text { Epsilon } \\
\text { Correction }\end{array}$ \\
Highlighting validity & 2 & 11023.42 & 5511.71 & 65.344 & .0000 & \\
$\quad$ Error & 58 & 4892.27 & 84.35 & & & .83 \\
Placement & 2 & 2032.22 & 1016.11 & 22.748 & .0000 & \\
$\quad$ Error & 58 & 2590.76 & 44.66 & & & .88 \\
Highlighting validity & & & & & & \\
X Placement & 4 & 239.99 & 59.99 & 1.188 & .3197 & \\
$\quad$ Error & 116 & 5857.53 & 50.50 & & & .66
\end{tabular}

ANOVA Summary Table 2

Accuracy. Target always present task x Highlighting validity (Valid, Invalid \& No) x Placement (Top, Middle \& Bottom) repeated measures ANOVA.

$\begin{array}{lrrrrrr}\text { Source of Variation } & \text { df } & \begin{array}{c}\text { Sum of } \\ \text { Squares }\end{array} & \begin{array}{c}\text { Mean } \\ \text { Square }\end{array} & \text { F } & \text { p } & \begin{array}{c}\text { Epsilon } \\ \text { Correction }\end{array} \\ \text { Highlighting validity } & 2 & 0.11 & 0.06 & 3.03 & .0560 & \\ \quad \text { Error } & 58 & 1.09 & 0.02 & & & .79 \\ \text { Placement } & 2 & 0.06 & 0.03 & 3.76 & .0291 & \\ \quad \text { Error } & 58 & 0.44 & 0.01 & & & .89 \\ \quad \text { Highlighting validity } & & & & & & \\ \quad \text { x Placement } & 4 & 0.02 & 0.01 & 0.93 & .4505 & .72 \\ \quad \text { Error } & 116 & 0.77 & 0.01 & & & \end{array}$


ANOVA Summary Table 3

Response Time. Target present/absent task x Highlighting validity (Valid, Invalid \& No) x Placement (Top, Middle $\&$ Bottom) repeated measures ANOVA.

\begin{tabular}{|c|c|c|c|c|c|c|}
\hline Source of Variation & df & $\begin{array}{l}\text { Sum of } \\
\text { Squares }\end{array}$ & $\begin{array}{l}\text { Mean } \\
\text { Square }\end{array}$ & $\mathrm{F}$ & $\mathrm{p}$ & $\begin{array}{c}\text { Epsilo } \\
n \\
\text { Correc } \\
\text { tion }\end{array}$ \\
\hline Highlighting validity & 2 & 4573.44 & 2286.72 & 157.45 & .0000 & \\
\hline Error & 58 & 813.33 & 14.52 & & & .91 \\
\hline Placement & 2 & 629.68 & 314.84 & 26.32 & .0000 & \\
\hline Error & 58 & 669.75 & 11.96 & & & .93 \\
\hline Highlighting validity $\mathrm{x}$ Placement & 4 & 91.78 & 22.95 & 2.80 & .0292 & \\
\hline Error & 116 & 917.18 & 8.19 & & & .94 \\
\hline
\end{tabular}

ANOVA Summary Table 4

Accuracy. Target present/absent task x Highlighting validity (Valid, Invalid \& No) x Placement (Top, Middle \& Bottom) repeated measures ANOVA.

$\begin{array}{lrrrrrr}\text { Source of Variation } & \text { df } & \begin{array}{c}\text { Sum of } \\ \text { Squares }\end{array} & \begin{array}{c}\text { Mean } \\ \text { Square }\end{array} & \text { F } & \text { p } & \begin{array}{c}\text { Epsilon } \\ \text { Correction }\end{array} \\ \quad \text { Highlighting validity } & 2 & 5.02 & 2.51 & 45.42 & .0000 & \\ \quad \text { Error } & 58 & 3.20 & 0.06 & & & .97 \\ \quad \text { Placement } & 2 & 0.71 & 0.35 & 9.03 & .0004 & \\ \quad \text { Error } & 58 & 2.26 & 0.04 & & & .96 \\ \quad \text { Highlighting validity x } & & & & & & \\ \quad \text { Placement } & 4 & 0.61 & 0.15 & 4.38 & .0024 & .84 \\ \quad \text { Error } & 116 & 4.00 & 0.04 & & & .84\end{array}$




\section{APPENDIX E}

Column Key

\section{Participant Data}

\section{Highlighting validity Placement Drug}

va $=$ Valid

$\mathrm{P} 1=$ Top

$\mathrm{a}=$ Advair

in = Invalid

$\mathrm{P} 2=$ Middle

$\mathrm{w}=$ Wellbutrin

no $=$ No highlighting

$\mathrm{P} 3=$ Bottom

\section{Target always present task - Response time data}

\begin{tabular}{|c|c|c|c|c|c|c|c|c|c|c|c|c|}
\hline & & & & & & & $\mathrm{aP} 2 \mathrm{w}$ & vaP2a & & & & vaP3a \\
\hline 1 & 2.604 & 1.883 & 5.278 & 1.912 & 2.283 & 9.935 & 1.592 & 1.723 & 11.096 & 14.862 & 19.619 & 13.770 \\
\hline 2 & 2.093 & 8.442 & 2.604 & 1.763 & & 4.296 & 43 & & & .850 & 852 & \\
\hline 3 & 824 & 2.38 & & & & 346 & 16 & 25 & & 225 & & 386 \\
\hline 4 & 109 & .406 & 6.000 & & 4.922 & 20.500 & 406 & 4.844 & 12.657 & 24.126 & 34 & 9.813 \\
\hline 5 & 000 & .719 & .235 & .953 & 1.922 & 137 & 2.297 & 2.016 & & & 953 & .859 \\
\hline 6 & 253 & 1 & .046 & & & & & 72 & & 08 & & \\
\hline 7 & 157 & .671 & & & & 16.328 & 60 & 2.704 & & 2.313 & & \\
\hline 8 & 84 & .8 & & & & & 94 & & & & & .843 \\
\hline 9 & 583 & .360 & .958 & & & & 492 & 33 & & & & \\
\hline 10 & 313 & 1.688 & & & & & 750 & 406 & & 14.688 & & 2.860 \\
\hline 1 & 750 & 2.18 & & & & & 84 & & & & & 282 \\
\hline 12 & 578 & 8.172 & 20.407 & 1.890 & 4.891 & 1.687 & 1.797 & 2.047 & 2.047 & 1.968 & & 2.640 \\
\hline 13 & 906 & 1.797 & & & 2.203 & & 234 & 2.016 & & 063 & & .500 \\
\hline 14 & 703 & 2.062 & 3.125 & 1.672 & 2.188 & & 390 & 2.141 & 984 & 21.704 & & 2.703 \\
\hline 15 & 154 & 3.004 & 3.375 & & 3.545 & 78 & 95 & 15 & & 434 & 64 & 160 \\
\hline 16 & 922 & 6.484 & 19.125 & 2.078 & 3.610 & & 203 & 2.000 & .297 & 15.609 & & .047 \\
\hline 17 & 193 & 1.963 & 4.056 & 2.043 & 2.574 & 327 & .003 & & & 34 & & 1.908 \\
\hline 18 & 14 & & & & & & & & & & & 08 \\
\hline 19 & 803 & 19.078 & 864 & 2.904 & 4.616 & 326 & 052 & 093 & 453 & 484 & 34 & 244 \\
\hline 20 & 625 & & & & & & & & & & & \\
\hline 21 & 535 & 3.064 & .485 & 2.855 & 3.114 & 18 & 3.325 & 3.485 & 7.681 & 5.868 & 943 & 34.209 \\
\hline 22 & 4.827 & 5.078 & 3.976 & 4.086 & 4.106 & 623 & 9.193 & 8.632 & 17.245 & 18.266 & 6.364 & 9.264 \\
\hline 23 & 4.346 & 4.016 & & 4.406 & 18.477 & 4.496 & 3.865 & & & 6.379 & & 4.687 \\
\hline 24 & .203 & 2.000 & 500 & 1.125 & 9.031 & 156 & 7.594 & 1.141 & 1.906 & .532 & 766 & 2.515 \\
\hline 25 & 2.938 & 2.843 & & 3.094 & 4.250 & 110 & 3.063 & 2.969 & & 12.000 & & 12.437 \\
\hline 26 & & 1 & & & & & & & & 15.282 & 033 & 12.558 \\
\hline 27 & 2.483 & 4.987 & 2.804 & 2.384 & 2.804 & 2.374 & 2.323 & 3.235 & 2.814 & 13.790 & 56.872 & 7.510 \\
\hline 28 & 2.934 & & & & & & 3.165 & 3.295 & 3.305 & 4.006 & 3.395 & 6.040 \\
\hline 29 & 2.864 & 3.686 & & 2.614 & & 2.994 & 3.786 & 3.816 & 11.967 & 4.977 & 12.127 & 4.677 \\
\hline 30 & 4.246 & 4.156 & 4.747 & 4.416 & 6.950 & 5.368 & 3.675 & 4.467 & 4.797 & 11.897 & 6.199 & 5.568 \\
\hline
\end{tabular}




\begin{tabular}{|c|c|c|c|c|c|c|c|c|c|c|c|}
\hline inP1w & inP1w & inP1a & inP1a & inP2w & inP2a & inP2a & inP2w & inP3a & inP3w & inP3a & inP3w \\
\hline 8.543 & 12.398 & 6.700 & 31.736 & 30.985 & 5.147 & 25.226 & 35.892 & 18.837 & 18.687 & 2.594 & 10.435 \\
\hline 2.173 & 4.637 & 11.397 & 5.758 & 3.685 & 13.759 & 6.038 & 5.548 & 28.781 & 8.432 & 6.349 & 11.276 \\
\hline 8.392 & 20.048 & 7.201 & 33.478 & 15.893 & 7.131 & 29.393 & 16.613 & 22.152 & 19.378 & 9.954 & 21.120 \\
\hline 4.313 & 6.781 & 15.219 & 5.125 & 19.688 & 7.734 & 66.344 & 19.953 & 41.390 & 15.579 & 27.015 & 17.719 \\
\hline 4.703 & 9.610 & 27.062 & 5.578 & 4.312 & 6.313 & 57.266 & 27.281 & 34.812 & 21.594 & 16.547 & 8.344 \\
\hline 4.777 & 40.699 & 9.354 & 12.538 & 18.527 & 17.585 & 30.664 & 7.150 & 41.649 & 23.364 & 20.349 & 33.428 \\
\hline 9.140 & 8.985 & 21.781 & 25.375 & 5.047 & 7.968 & 15.609 & 8.844 & 31.047 & 17.500 & 35.938 & 15.734 \\
\hline 6.000 & 26.938 & 5.843 & 154.578 & 16.375 & 18.438 & 19.719 & 15.734 & 21.344 & 21.984 & 22.594 & 20.078 \\
\hline 13.890 & 15.212 & 6.589 & 29.382 & 6.519 & 6.048 & 13.579 & 6.369 & 20.911 & 46.678 & 19.788 & 14.721 \\
\hline 20.625 & 16.203 & 19.000 & 29.406 & 8.969 & 4.985 & 19.594 & 5.453 & 27.531 & 26.625 & 20.984 & 13.610 \\
\hline 6.454 & 19.500 & 7.422 & 30.281 & 18.297 & 6.297 & 9.859 & 7.079 & 17.610 & 40.906 & 5.797 & 4.375 \\
\hline 6.594 & 15.000 & 9.703 & 40.469 & 3.844 & 6.094 & 29.235 & 13.438 & 46.485 & 29.532 & 30.797 & 8.718 \\
\hline 5.235 & 7.016 & 4.125 & 11.109 & 28.609 & 6.344 & 31.875 & 41.781 & 13.937 & 17.797 & 8.750 & 23.657 \\
\hline 33.406 & 26.141 & 4.516 & 8.265 & 11.375 & 4.828 & 8.265 & 3.110 & 15.375 & 8.859 & 7.312 & 17.906 \\
\hline 3.775 & 3.575 & 7.701 & 25.096 & 11.587 & 4.607 & 17.605 & 59.295 & 27.710 & 57.573 & 12.038 & 10.225 \\
\hline 61.031 & 27.078 & 58.657 & 39.109 & 13.281 & 71.546 & 22.625 & 53.641 & 15.047 & 92.078 & 38.093 & 28.532 \\
\hline 59.956 & 9.244 & 13.249 & 4.766 & 16.453 & 4.817 & 62.009 & 16.654 & 21.872 & 18.347 & 17.215 & 31.716 \\
\hline 14.711 & 9.674 & 24.205 & 40.759 & 25.176 & 11.887 & 26.979 & 13.690 & 15.382 & 21.400 & 26.248 & 24.185 \\
\hline 41.019 & 21.431 & 3.795 & 5.938 & 16.424 & 5.548 & 9.514 & 5.989 & 12.668 & 16.613 & 8.592 & 11.046 \\
\hline 6.094 & 12.156 & 5.781 & 32.125 & 9.265 & 20.718 & 22.687 & 11.046 & 20.625 & 13.016 & 10.532 & 11.000 \\
\hline 19.058 & 25.647 & 8.893 & 14.490 & 24.205 & 8.042 & 40.228 & 16.434 & 13.570 & 41.590 & 61.559 & 21.190 \\
\hline 6.830 & 8.792 & 4.887 & 9.214 & 10.035 & 5.117 & 35.321 & 36.622 & 11.567 & 33.187 & 16.524 & 16.994 \\
\hline 7.451 & 30.574 & 8.573 & 9.023 & 13.760 & 22.292 & 17.986 & 8.231 & 7.731 & 19.208 & 15.342 & 45.064 \\
\hline 7.328 & 17.265 & 25.890 & 28.844 & 11.750 & 13.906 & 28.610 & 20.031 & 25.813 & 22.640 & 17.062 & 13.969 \\
\hline 7.422 & 10.203 & 25.953 & 23.265 & 10.750 & 5.735 & 11.922 & 6.921 & 43.531 & 13.672 & 15.328 & 11.609 \\
\hline 5.047 & 17.495 & 6.539 & 25.287 & 39.307 & 12.408 & 24.575 & 14.791 & 10.805 & 14.611 & 12.668 & 5.458 \\
\hline 5.548 & 36.483 & 82.729 & 10.425 & 14.191 & 8.182 & 22.813 & 23.194 & 17.034 & 39.316 & 19.198 & 18.036 \\
\hline 14.002 & 34.553 & 7.311 & 49.337 & 40.182 & 13.360 & 6.951 & 6.631 & 26.000 & 9.475 & 5.779 & 10.266 \\
\hline 20.079 & 10.315 & 5.488 & 52.085 & 7.931 & 7.921 & 18.227 & 10.124 & 10.875 & 38.155 & 10.385 & 6.880 \\
\hline 18.967 & 23.223 & 9.684 & 10.975 & 53.627 & 10.125 & 12.778 & 20.549 & 15.823 & 25.827 & 20.850 & 141.263 \\
\hline
\end{tabular}




\begin{tabular}{|c|c|c|c|c|c|c|c|c|c|c|c|}
\hline oP1a & oP1w & oP1a & noP1w & noP2a & noP2a & & noP2w & & & & \\
\hline 50.753 & 7.780 & .486 & 7.411 & 3.114 & & 2.058 & 2.463 & .506 & & 2.913 & .122 \\
\hline 14.721 & 2.434 & 9.018 & 5.558 & 31.916 & & & .111 & & & & 904 \\
\hline 5.958 & 35 & .264 & .534 & & & & & & & & 880 \\
\hline 6.922 & 188 & .438 & 06 & 29.437 & & 1.235 & 235 & & 360 & 0.219 & 5.312 \\
\hline 27.687 & 2.844 & .031 & 14. & 3.922 & 1.563 & & .000 & & & & 10.937 \\
\hline 9.805 & 1.920 & .775 & & & & & 300 & & & & 4.977 \\
\hline 9.125 & .156 & .187 & .312 & & & & & & & & .281 \\
\hline 22.687 & .328 & 12.000 & 86.094 & 15.750 & 572 & 1.531 & .735 & 031 & 4.813 & 5.640 & 25.765 \\
\hline 23.123 & 29.432 & .815 & 15.302 & .565 & & & & & & & 2.354 \\
\hline 19.484 & . 719 & 1.781 & & 26.141 & 125 & & 688 & & 531 & 0.985 & 7.531 \\
\hline 6.609 & 20.000 & & & & & & & & & & 625 \\
\hline 7.219 & 0.344 & & & & & & & & & .875 & 9.000 \\
\hline 30.485 & 2.141 & 7.390 & 11.797 & 17.922 & & 8.015 & 703 & & & 406 & .969 \\
\hline 49.297 & 093 & 5.28 & & 17.266 & 26.266 & & 797 & & & & .781 \\
\hline 16.233 & 16.434 & 59 & & & & & & & & & .035 \\
\hline 11.359 & 21.344 & 1.828 & & 16.516 & 24.719 & 76.625 & 2.563 & 797 & 844 & 203 & 69.454 \\
\hline 8.973 & 14.210 & 5.963 & 7.221 & 38.366 & & & & & & & 25.877 \\
\hline 5.368 & & & & 13. & & & & & & & 3.927 \\
\hline 24.695 & 3.18 & 0.395 & & & & & 295 & & & & .196 \\
\hline 5.453 & 3.844 & & & & & & & & & & 21.766 \\
\hline 306 & 8.873 & & & 10.155 & & 66.246 & 24.966 & & 4.822 & 17.075 & 6.669 \\
\hline 6.319 & 4.666 & 8.762 & 13.920 & 6.830 & 9.945 & 12.848 & 2.507 & & 1.020 & 8.106 & 14.251 \\
\hline 15.352 & 19.879 & & & & & & & & & & .586 \\
\hline 9.797 & 2.438 & 25.484 & 14.969 & 4.016 & 23.141 & 5.500 & 31.235 & 22.125 & 12.594 & 18.266 & 27.657 \\
\hline 3.250 & 15.515 & 26.406 & & & & & & & & 21.422 & 13.203 \\
\hline 4.346 & 38.846 & & & & & & 9.964 & & & 17.405 & 5.788 \\
\hline 56.001 & 16.293 & 5.888 & 12.919 & 11.537 & 47.479 & 237.322 & 13.690 & 145.479 & 107.454 & 47.118 & 18.677 \\
\hline 8.083 & 18.388 & 4.567 & & 11.949 & 12.049 & & 13.921 & 6.010 & 13.401 & 8.764 & 5.879 \\
\hline 53.217 & 2.568 & & & & & & & & 56.341 & 10.104 & 16.724 \\
\hline 21.501 & 31.585 & 14.060 & 5.298 & 12.078 & 101.666 & 16.984 & 11.537 & 70.722 & 28.831 & 18.727 & 27.840 \\
\hline
\end{tabular}


Target always present task - Accuracy data

\begin{tabular}{|c|c|c|c|c|c|c|c|c|c|c|c|c|}
\hline subject & vaP1a & vaP1a & vaP1w & vaP1w & $\mathrm{vaP} 2 \mathrm{a}$ & $\mathrm{vaP} 2 \mathrm{w}$ & $\mathrm{vaP} 2 \mathrm{w}$ & $\mathrm{vaP} 2 \mathrm{a}$ & vaP3w & vaP3a & vaP3w & vaP3a \\
\hline 1 & 1 & 1 & 1 & 1 & 0 & 1 & 1 & 1 & 1 & 1 & 1 & 1 \\
\hline 2 & 1 & 1 & 1 & 1 & 1 & 1 & 1 & 1 & 1 & 1 & 1 & 1 \\
\hline 3 & 1 & 1 & 1 & 1 & 1 & 1 & 1 & 1 & 1 & 1 & 1 & 1 \\
\hline 4 & 1 & 1 & 1 & 1 & 1 & 1 & 1 & 1 & 1 & 1 & 1 & 1 \\
\hline 5 & 1 & 1 & 1 & 1 & 1 & 1 & 1 & 1 & 1 & 1 & 1 & 1 \\
\hline 6 & 1 & 1 & 1 & 1 & 1 & 1 & 1 & 1 & 1 & 1 & 1 & 1 \\
\hline 7 & 1 & 1 & 1 & 0 & 1 & 1 & 1 & 1 & 1 & 1 & 1 & 1 \\
\hline 8 & 1 & 1 & 1 & 1 & 1 & 1 & 1 & 1 & 1 & 0 & 1 & 1 \\
\hline 9 & 1 & 1 & 1 & 1 & 1 & 1 & 1 & 1 & 1 & 1 & 1 & 1 \\
\hline 10 & 1 & 1 & 1 & 1 & 1 & 1 & 1 & 1 & 1 & 1 & 1 & 1 \\
\hline 11 & 1 & 1 & 1 & 1 & 1 & 1 & 1 & 1 & 1 & 1 & 1 & 1 \\
\hline 12 & 1 & 1 & 1 & 1 & 1 & 1 & 1 & 1 & 1 & 0 & 1 & 1 \\
\hline 13 & 1 & 1 & 1 & 1 & 1 & 1 & 1 & 1 & 1 & 1 & 1 & 1 \\
\hline 14 & 1 & 1 & 1 & 1 & 1 & 1 & 1 & 1 & 1 & 1 & 1 & 1 \\
\hline 15 & 1 & 1 & 1 & 1 & 1 & 1 & 1 & 1 & 1 & 1 & 1 & 1 \\
\hline 16 & 1 & 1 & 1 & 1 & 1 & 1 & 1 & 1 & 1 & 1 & 1 & 1 \\
\hline 17 & 1 & 1 & 1 & 1 & 1 & 1 & 1 & 1 & 1 & 1 & 1 & 1 \\
\hline 18 & 1 & 1 & 1 & 1 & 1 & 1 & 1 & 1 & 1 & 1 & 1 & 1 \\
\hline 19 & 1 & 1 & 1 & 1 & 1 & 1 & 1 & 1 & 1 & 1 & 1 & 1 \\
\hline 20 & 1 & 1 & 1 & 1 & 1 & 1 & 1 & 1 & 1 & 1 & 1 & 1 \\
\hline 21 & 1 & 1 & 1 & 1 & 1 & 1 & 1 & 1 & 1 & 1 & 1 & 1 \\
\hline 22 & 1 & 1 & 1 & 1 & 1 & 1 & 1 & 0 & 1 & 1 & 1 & 1 \\
\hline 23 & 1 & 1 & 1 & 1 & 1 & 1 & 1 & 1 & 1 & 1 & 1 & 1 \\
\hline 24 & 1 & 0 & 0 & 0 & 1 & 1 & 1 & 0 & 0 & 0 & 0 & 0 \\
\hline 25 & 1 & 1 & 1 & 1 & 1 & 1 & 1 & 1 & 1 & 0 & 1 & 1 \\
\hline 26 & 1 & 1 & 1 & 1 & 1 & 1 & 1 & 1 & 1 & 1 & 1 & 1 \\
\hline 27 & 1 & 1 & 0 & 1 & 1 & 1 & 1 & 0 & 1 & 1 & 1 & 0 \\
\hline 28 & 1 & 1 & 1 & 1 & 1 & 1 & 1 & 1 & 1 & 1 & 1 & 1 \\
\hline 29 & 1 & 1 & 1 & 1 & 1 & 1 & 1 & 1 & 1 & 1 & 1 & 1 \\
\hline 30 & 1 & 1 & 1 & 1 & 1 & 1 & 1 & 1 & 1 & 1 & 1 & 1 \\
\hline
\end{tabular}




\begin{tabular}{|c|c|c|c|c|c|c|c|c|c|c|c|}
\hline inP1w & inP1w & inP1a & inP1a & $\mathrm{inP} 2 \mathrm{w}$ & inP2a & inP2a & $\mathrm{inP} 2 \mathrm{w}$ & inP3a & inP3w & inP3a & inP3w \\
\hline 1 & 1 & 1 & 1 & 1 & 1 & 1 & 1 & 1 & 1 & 1 & 1 \\
\hline 1 & 1 & 1 & 1 & 1 & 1 & 1 & 1 & 1 & 1 & 1 & 1 \\
\hline 0 & 1 & 1 & 1 & 1 & 1 & 1 & 1 & 1 & 1 & 1 & 1 \\
\hline 1 & 1 & 1 & 1 & 1 & 1 & 1 & 1 & 1 & 1 & 1 & 1 \\
\hline 1 & 1 & 1 & 1 & 1 & 1 & 1 & 1 & 1 & 1 & 1 & 1 \\
\hline 1 & 1 & 1 & 1 & 1 & 1 & 1 & 1 & 1 & 1 & 1 & 1 \\
\hline 0 & 1 & 1 & 1 & 0 & 1 & 1 & 0 & 1 & 1 & 1 & 0 \\
\hline 1 & 1 & 1 & 1 & 1 & 1 & 1 & 1 & 1 & 1 & 1 & 1 \\
\hline 1 & 1 & 1 & 1 & 1 & 1 & 1 & 0 & 1 & 1 & 1 & 1 \\
\hline 1 & 1 & 1 & 1 & 1 & 1 & 1 & 1 & 1 & 1 & 1 & 1 \\
\hline 1 & 1 & 1 & 1 & 1 & 1 & 1 & 1 & 1 & 1 & 1 & 1 \\
\hline 1 & 1 & 1 & 1 & 1 & 1 & 1 & 1 & 0 & 1 & 1 & 1 \\
\hline 1 & 1 & 1 & 1 & 1 & 1 & 1 & 1 & 1 & 1 & 1 & 1 \\
\hline 1 & 1 & 1 & 1 & 1 & 1 & 1 & 1 & 1 & 1 & 1 & 1 \\
\hline 1 & 1 & 1 & 1 & 0 & 1 & 1 & 1 & 1 & 1 & 1 & 1 \\
\hline 1 & 1 & 1 & 1 & 1 & 1 & 1 & 1 & 1 & 1 & 1 & 1 \\
\hline 0 & 1 & 1 & 1 & 1 & 1 & 1 & 1 & 1 & 1 & 1 & 1 \\
\hline 1 & 1 & 1 & 1 & 1 & 1 & 1 & 1 & 1 & 1 & 1 & 1 \\
\hline 1 & 1 & 1 & 1 & 0 & 1 & 1 & 1 & 1 & 1 & 1 & 1 \\
\hline 1 & 0 & 1 & 1 & 1 & 1 & 0 & 1 & 1 & 1 & 1 & 1 \\
\hline 0 & 1 & 1 & 1 & 1 & 1 & 1 & 1 & 1 & 1 & 1 & 1 \\
\hline 1 & 1 & 1 & 1 & 1 & 1 & 1 & 1 & 1 & 1 & 1 & 1 \\
\hline 1 & 1 & 1 & 1 & 1 & 1 & 1 & 1 & 1 & 1 & 1 & 1 \\
\hline 1 & 0 & 1 & 1 & 1 & 1 & 0 & 1 & 1 & 1 & 1 & 0 \\
\hline 1 & 1 & 1 & 1 & 1 & 1 & 1 & 1 & 1 & 1 & 1 & 1 \\
\hline 1 & 1 & 1 & 1 & 1 & 1 & 1 & 1 & 1 & 1 & 1 & 1 \\
\hline 1 & 1 & 1 & 1 & 1 & 1 & 1 & 1 & 0 & 1 & 1 & 1 \\
\hline 1 & 1 & 0 & 1 & 1 & 1 & 1 & 0 & 1 & 1 & 1 & 1 \\
\hline 1 & 1 & 1 & 1 & 1 & 1 & 1 & 1 & 1 & 1 & 0 & 1 \\
\hline 1 & 1 & 1 & 1 & 1 & 1 & 1 & 1 & 1 & 1 & 1 & 1 \\
\hline
\end{tabular}




$\begin{array}{cccccccccccc}\text { noP1a } & \text { noP1w } & \text { noP1a } & \text { noP1w } & \text { noP2a } & \text { noP2a } & \text { noP2w } & \text { noP2w } & \text { noP3w } & \text { noP3a } & \text { noP3w } & \text { noP3a } \\ 1 & 1 & 1 & 1 & 1 & 1 & 1 & 1 & 1 & 1 & 1 & 1 \\ 1 & 1 & 1 & 1 & 1 & 1 & 1 & 1 & 1 & 1 & 1 & 1 \\ 1 & 1 & 1 & 1 & 1 & 1 & 1 & 1 & 1 & 1 & 1 & 1 \\ 1 & 1 & 1 & 1 & 1 & 1 & 1 & 1 & 1 & 1 & 1 & 1 \\ 1 & 1 & 1 & 1 & 1 & 1 & 1 & 1 & 1 & 1 & 1 & 1 \\ 1 & 1 & 1 & 1 & 1 & 1 & 1 & 1 & 1 & 1 & 1 & 1 \\ 1 & 1 & 1 & 1 & 1 & 0 & 1 & 1 & 1 & 1 & 1 & 1 \\ 1 & 1 & 1 & 1 & 1 & 1 & 1 & 1 & 1 & 1 & 1 & 1 \\ 1 & 1 & 1 & 1 & 1 & 1 & 1 & 1 & 1 & 1 & 1 & 1 \\ 1 & 1 & 1 & 1 & 1 & 1 & 1 & 1 & 1 & 1 & 1 & 0 \\ 1 & 1 & 1 & 1 & 1 & 1 & 1 & 1 & 1 & 1 & 1 & 0 \\ 1 & 1 & 1 & 1 & 1 & 1 & 1 & 1 & 1 & 1 & 1 & 1 \\ 1 & 1 & 1 & 1 & 1 & 1 & 1 & 1 & 1 & 1 & 1 & 1 \\ 1 & 1 & 1 & 1 & 1 & 1 & 1 & 1 & 1 & 1 & 1 & 1 \\ 1 & 1 & 1 & 1 & 1 & 1 & 1 & 1 & 1 & 1 & 1 & 1 \\ 1 & 1 & 1 & 1 & 1 & 1 & 1 & 1 & 1 & 1 & 1 & 1 \\ 1 & 1 & 1 & 1 & 1 & 1 & 1 & 1 & 1 & 1 & 1 & 1 \\ 1 & 1 & 1 & 1 & 1 & 1 & 1 & 1 & 1 & 1 & 1 & 1 \\ 1 & 1 & 1 & 1 & 1 & 1 & 1 & 1 & 1 & 1 & 1 & 1 \\ 1 & 1 & 1 & 1 & 1 & 1 & 1 & 1 & 1 & 1 & 1 & 1 \\ 1 & 1 & 1 & 1 & 1 & 1 & 1 & 1 & 1 & 1 & 1 & 1 \\ 1 & 1 & 1 & 1 & 1 & 1 & 1 & 1 & 1 & 1 & 1 & 1 \\ 1 & 1 & 1 & 1 & 1 & 1 & 1 & 1 & 1 & 1 & 1 & 1 \\ 1 & 1 & 1 & 1 & 1 & 1 & 1 & 1 & 1 & 1 & 1 & 1 \\ 1 & 1 & 1 & 1 & 1 & 1 & 1 & 1 & 1 & 1 & 1 & 1 \\ 1 & 1 & 1 & 1 & 1 & 1 & 1 & 1 & 1 & 1 & 1 & 1 \\ 1 & 1 & 1 & 1 & 1 & 1 & 1 & 1 & 1 & 1 & 1 & 1 \\ 1 & 1 & 1 & 1 & 1 & 1 & 1 & 1 & 1 & 1 & 1 & 1 \\ 1 & 1 & 1 & 1 & 1 & 1 & 1 & 1 & 1 & 1 & 1 & 1 \\ 1 & 1 & 1 & 1 & 1 & 1 & 1 & 1 & 1 & 1 & 1 & 1\end{array}$




\section{Target always present task-Demographic data}

$\begin{array}{cc}\text { gender } & \text { age } \\ \text { f } & 18 \\ \text { f } & 18 \\ \text { m } & 19 \\ \text { m } & 19 \\ \text { m } & 18 \\ \text { m } & 19 \\ \text { f } & 19 \\ \text { f } & 18 \\ \text { m } & 19 \\ \text { m } & 20 \\ \text { m } & 42 \\ \text { m } & 19 \\ \text { f } & 18 \\ \text { f } & 18 \\ \text { m } & 19 \\ \text { f } & 33 \\ \text { f } & 57 \\ \text { m } & 38 \\ \text { m } & 25 \\ \text { m } & 34 \\ \text { f } & 35 \\ \text { f } & 29 \\ \text { f } & 46 \\ \text { m } & 28 \\ \text { f } & 39 \\ \text { f } & 53 \\ \text { m } & 40 \\ \text { m } & 33 \\ \text { m } & 32\end{array}$

race
caucasian
caucasian
african
hispanic
caucasian
caucasian
caucasian
hispanic
middleeast
caucasian
multiracial
asian
caucasian
multiracial
caucasian
caucasian
caucasian
caucasian
hispanic
caucasian
caucasian
asian
caucasian
hispanic
caucasian
caucasian
multiracial
caucasian

student
$\mathrm{y}$
$\mathrm{y}$
$\mathrm{y}$
$\mathrm{y}$
$\mathrm{y}$
$\mathrm{y}$
$\mathrm{y}$
$\mathrm{y}$
$\mathrm{y}$
$\mathrm{y}$
$\mathrm{y}$
$\mathrm{y}$
$\mathrm{y}$
$\mathrm{y}$
$\mathrm{y}$
$\mathrm{n}$
$\mathrm{n}$
$\mathrm{n}$
$\mathrm{n}$
$\mathrm{n}$
$\mathrm{n}$
$\mathrm{n}$
$\mathrm{n}$
$\mathrm{n}$
$\mathrm{n}$
$\mathrm{n}$
$\mathrm{n}$
$\mathrm{n}$

college

comp_usage surfing shopping surf_freq

fresh

fresh

soph

soph

fresh

fresh

fresh

fresh

soph

soph

senior

fresh

fresh

fresh

soph

\begin{tabular}{l}
4 \\
3 \\
5 \\
5 \\
5 \\
4 \\
4 \\
4 \\
4 \\
5 \\
5 \\
4 \\
3 \\
3 \\
4 \\
4 \\
5 \\
5 \\
4 \\
5 \\
4 \\
5 \\
5 \\
5 \\
4 \\
5 \\
5 \\
5 \\
\hline
\end{tabular}

$\begin{array}{lll}4 & 5 & 4 \\ 5 & 2 & 5 \\ 5 & 4 & 5 \\ 5 & 2 & 5 \\ 5 & 3 & 5 \\ 4 & 4 & 5 \\ 5 & 3 & 5 \\ 3 & 2 & 3 \\ 4 & 3 & 5 \\ 5 & 4 & 5 \\ 5 & 5 & 3 \\ 4 & 1 & 5 \\ 4 & 3 & 5 \\ 4 & 2 & 5 \\ 4 & 2 & 5 \\ 4 & 4 & 5 \\ 5 & 4 & 5 \\ 4 & 4 & 5 \\ 4 & 4 & 5 \\ 5 & 5 & 5 \\ 4 & 5 & 5 \\ 5 & 4 & 5 \\ 5 & 3 & 5 \\ 5 & 4 & 5 \\ 4 & 3 & 5 \\ 5 & 5 & 5 \\ 5 & 3 & 5 \\ 5 & 5 & 5 \\ & & \end{array}$

